\title{
Noise regularization and computations for the 1-dimensional stochastic Allen-Cahn problem
}

\author{
MARKOS A. KATSOULAKIS ${ }^{\dagger}$ \\ Department of Mathematics and Statistics, University of Massachusetts, \\ Amherst, MA 01003, USA \\ GEORGIOS T. KOSSIORIS \\ Department of Mathematics, University of Crete, \\ Knossos Avenue, Heraklion, GR-71409 Greece, and \\ Institute for Applied and Computational Mathematics, \\ Foundation for Research and Technology-Hellas, \\ Vasilika Vouton, Crete GR-71110, Greece \\ AND \\ OMAR LAKKIS ${ }^{\S}$ \\ Department of Mathematics, University of Sussex, \\ Brighton, BN1 9RF, United Kingdom
}

[Received 19 July 2005 and in revised form 16 September 2006]

\begin{abstract}
We address the numerical discretization of the Allen-Cahn problem with additive white noise in one-dimensional space. Our main focus is to understand the behavior of the discretized equation with respect to a small "interface thickness" parameter and the noise intensity. The discretization is conducted in two stages: (1) regularize the white noise and study the regularized problem, (2) approximate the regularized problem. We address (1) by introducing a piecewise constant random approximation of the white noise with respect to a space-time mesh. We analyze the regularized problem and study its relation to both the original problem and the deterministic Allen-Cahn problem. Step (2) is then performed leading to a practical Monte Carlo method combined with a finite element-implicit Euler scheme. The resulting numerical scheme is tested against theoretical benchmark results concerning the behavior of the solution as the interface thickness goes to zero.
\end{abstract}

\section{Introduction}

Stochastic partial differential equation (SPDE) models arise in numerous applications ranging from materials science, surface processes and macromolecular dynamics [Co070, Spo89], to atmosphere and ocean modeling [LN03] and epidemiology [Dur99]. These models are typically derived from finer and more detailed models where unresolved degrees of freedom are represented by suitable stochastic forcing terms. There are also some notable rigorous derivations from microscopic scales in special asymptotic regimes, e.g. [BPRS93, MT95].

\footnotetext{
${ }^{\dagger}$ E-mail: markos@math.umass.edu

E-mail: kosioris@math.uoc.gr

${ }^{\S}$ E-mail: o.lakkis@sussex.ac.uk
} 
An important class of models consists of the stochastic Ginzburg-Landau models which are typically obtained from microscopic lattice models for a suitable order parameter (e.g., spin), by statistical mechanics renormalization arguments combined with detailed balance laws.

Numerical simulation of these nonlinear SPDE's constitutes an important research issue. On the practical side, one is interested in having efficient, reliable and not too complex numerical codes which can be used either in the context of Monte Carlo methods or for sample paths simulations of the physical models of phase transition, e.g. [WB95, KM99, KK01, Sha00]. From a more theoretical view-point, understanding the issues arising from the discretization of SPDE's, in a more general setting than phase separation, both through finite difference or finite element schemes, turns out to be a non-obvious departure from numerical schemes for deterministic models ANZ98, Gyö99. DZ02, BTZ04, ST03].

In this paper, our focus is on the numerical simulation of the stochastic Allen-Cahn problem, which is one of the simplest models exhibiting the phenomena of interface formation and nucleation. The stochastic Allen-Cahn problem is an ad-hoc white noise perturbation of the deterministic Allen-Cahn, given by

$$
\partial_{t} u(x, t)-\partial_{x x} u(x, t)+f_{\epsilon}(u(x, t))=\epsilon^{\gamma} \partial_{x t} W(x, t) \quad \text { for } x \in D, t \in[0, \infty),
$$

where $D=(-1,1) \subset \mathbb{R}^{1}, \epsilon>0$ and $f_{\epsilon}$ is an odd nonlinearity scaled by $1 / \epsilon^{2}$ and $\partial_{x t} W$ is the space-time white noise (see $\$ 2.1$ for the details). This is a stochastic version of the well-known deterministic Allen-Cahn problem describing the evolution in time of a polycrystalline material [AC79]. We take boundary conditions of Neumann type and the initial condition to be a resolved profile; we refer again to $\$ 2$ for the details. Note that this equation, with white noise, is tractable only in one spatial dimension, which is the case we will study. In higher space dimensions, one has to consider noise which is colored in space.

Equation (1.1) is a type A model in Halperin's classification [HH77]. It is non-conservative in the order parameter $u$ and exhibits both nucleation and interface formation, whilst retaining a relatively simple structure without multiplicative or conservative noise terms encountered in type B models, such as the Cahn-Hilliard-Cook equation [KM99].

While a thorough discussion of 1.1 is given in $\$ 2$ it is worth mentioning here that this SPDE, with the white noise term, is well-posed only in one space dimension. Two important pieces of work concerned with the analytic and probabilistic aspects of (1.1) are those of Funaki [Fun95] and Brassesco, De Masi \& Presutti [BDMP95]. In both papers, the authors study the asymptotic behavior of the solution processes as $\epsilon \rightarrow 0$. In particular, it turns out that, under suitable time-space rescaling, the solution with initial value taken to be (roughly speaking) a step function, converges (in an appropriate probabilistic sense) to the step function with its jump point performing a Brownian motion.

Though finite difference schemes have been used for simulations [KM99, KK01], we follow here a finite element approach. The reason driving us to understand finite element methods (FEM) for such equations is that FEM constitute a quite flexible tool, especially for problems in higher dimensions where one may have to deal with complex geometries. Also, finite elements are naturally suited for adaptive schemes where fine scales may be resolved only on small portions of the domain in order to obtain a reasonable accuracy. We believe that understanding the FEM in a nonadaptive one-dimensional setting will pave the way to more sophisticated studies.

Our strategy to formulate a finite element scheme for (1.1) follows an idea introduced for linear problems by Allen, Novosel \& Zhang [ANZ98, and consists in two steps: 
1. regularize the noise term $\partial_{x t} W$, by replacing it with a somewhat smoother approximate white noise $\partial_{x t} \bar{W}$;

2. discretize the regularized problem.

This approach allows us to conduct a rigorous analysis of the approximation. It makes the subsequent finite element discretization straightforward. Note that a finite difference variant based on our regularization is also possible.

Our first task, carried out in $\$ 3$, is to construct a regularization, denoted $\partial_{x t} \bar{W}(x, t)$, of $\partial_{x t} W(x, t)$ (appearing in 11.1 ) with respect to an underlying uniform partition, $\mathscr{D}_{\sigma} \times \mathscr{I}_{\rho}$, of the space-time domain $D \times I$. In the spirit of FEM, this regularization process consists of a projection of the white noise onto an appropriate space of piecewise constant space-time functions, which may be viewed as the mixed derivatives of hat functions. This idea, which has been successfully used in the context of the linear heat equation [ANZ98], leads to the regularized problem

$$
\partial_{t} u(x, t)-\partial_{x x} u(x, t)+f_{\epsilon}(u(x, t))=\epsilon^{\gamma} \partial_{x t} \bar{W}(x, t) \quad \text { for } x \in D, t \in[0, \infty) .
$$

Notice that $\partial_{x t} \bar{W}$ is still a stochastic process in space-time, but it is much smoother than white noise, which allows equation (1.2) to be interpreted in the usual PDE sense pathwise.

In $\$ 4$ after recalling some basic properties of problem 1.2 and its solution, we prove Theorem 4.4, which states that the solution of the regularized problem converges - in an appropriate senseto the solution of the original SPDE (1.1) as the space-time partition becomes infinitely fine.

Next, in $\$ 5$, we relate the solution of the regularized problem to the deterministic solution of the Allen-Cahn equation. Our main result here, Theorem 5.4, proved for $\gamma>3$, indicates that the regularization parameters have to be sufficiently small for the noise to be captured in the numerical computations. In fact, according to this theorem the weaker the noise, the finer space-time mesh one must take in order to see the noise effects. This is due to the fact that for a fixed space-time mesh and $\epsilon \rightarrow 0$, the distance between the regularized stochastic solution $\bar{u}$ and the deterministic solution, $q$, is of higher order in $\epsilon$ than the distance between $\bar{u}$ and $u$. Our proof makes use of the spectrum estimates of the linearized elliptic differential operator $-\partial_{x x}+f_{\epsilon}^{\prime}(q)$, derived independently by Xinfu Chen [Che94] and de Mottoni \& Schatzman [dMS95].

We note that while numerical schemes for the stochastic Allen-Cahn involving a spectral approach to white noise have been analyzed [Liu03], this is, up to our knowledge, a first analysis using projection methods to regularize the white noise.

Step 2 of our strategy is accomplished in $\$ 6$, where we derive a simple finite element scheme for the regularized problem (1.2). This is a scheme which uses piecewise polynomial finite elements to discretize the space variable and an implicit (backward) Euler scheme to discretize the time variable. Related numerical schemes have been thoroughly analyzed and successfully applied in the context of the deterministic Allen-Cahn problem [FP03, KNS04, FW05] and for the stochastic linear heat diffusion problem [ANZ98]. It is for the first time, up to our knowledge, that this scheme is employed in a stochastic and nonlinear setting. The issues of regularity of the regularized solution and the convergence of the FEM are objects of our current research.

In \$7, we test our scheme in combination with a Monte Carlo simulation. The test consists in reconciling the computational results with the theoretical results obtained by Funaki [Fun95] and Brassesco, De Masi \& Presutti [BDMP95] independently. Our benchmarking procedure consists in tracking the so-called center of a resolved profile of the Allen-Cahn equation as time evolves, performing statistics thereon and comparing them with the probabilistic results coming from the theory. The following conclusions are drawn: (1) The robustness of the Monte Carlo method 
depends on the noise intensity; the lower the noise, the higher the observed robustness. (2) The noise has to be resolved satisfactorily in order to see stochastic effects. In contrast with the first conclusion, the lower the noise, the more one has to resolve the mesh in order to see the noise. This is in competition with the need to have a fine mesh in order to resolve the transition layer, due to the structure of the solution of the Allen-Cahn equation. (3) The behavior captured by the numerics is consistent with the theoretical results; in particular, the Mueller-Funaki time scale $1+2 \gamma$ (see 5.6 for the details) and the corresponding Brownian motion diffusion coefficient are clearly exhibited by our numerical results. We close with some computations that capture the drift of the interface, modeled by the Allen-Cahn equation. This drift, typical of the stochastic solution, is quite fast with respect to the deterministic case where the solutions are metastable states.

\section{Set up}

\section{$2.1 \quad$ Noisy Allen-Cahn problem}

We will study an initial-boundary value problem associated with the semilinear parabolic partial differential equation with additive white noise, known as the stochastic (or noisy) Allen-Cahn equation given by (1.1). The nonlinearity $f_{\epsilon}$ is the derivative of an even coercive function $F_{\epsilon}$ with exactly two minimum points. A function such as $F_{\epsilon}$ is known as a double-well potential and, for sake of conciseness, we focus on the model potential explicitly defined by

$$
F_{\epsilon}(\xi)=\frac{1}{4 \epsilon^{2}}\left(\xi^{2}-1\right)^{2} \quad \text { for } \xi \in \mathbb{R} .
$$

Here $\epsilon \in \mathbb{R}^{+}$is a scaling parameter. The term $\partial_{x t} W$ is the space-time Gaussian white noise, which can be defined as the mixed distributional derivative of a Brownian sheet $W$ [Wal86, KX95]. The parameter $\gamma \in \mathbb{R}$ models the intensity of the white noise and plays a delicate role in the analysis, as $\epsilon \rightarrow 0$.

The presence of the right-hand side makes (1.1) a randomly perturbed version of the Allen-Cahn equation which is a stochastic PDE (SPDE). A solution of such an equation has to be interpreted in the stochastic sense. That is, for each $t$, the solution $u(\cdot, t)$ is understood as a random process on an underlying probability measure space $(\Omega, \mathscr{F}, P)$ with values in a suitable function space defined on $D$. Equation (1.1), supplemented with the initial condition

$$
u(x, 0)=u_{0}(x), \quad \forall x \in D,
$$

and with the Neumann boundary conditions

$$
\partial_{x} u(-1, t)=\partial_{x} u(1, t)=0, \quad \forall t \in \mathbb{R}^{+},
$$

defines the stochastic Allen-Cahn problem. For simplicity, we assume that the initial condition $u_{0}$ is smooth enough and satisfies the boundary conditions. In $\$ 5$ we shall focus on a more particular class of initial conditions known as resolved profiles.

\subsection{Space-time stochastic integral}

One can give a mathematically rigorous definition of a solution of the stochastic Allen-Cahn problem [1.1], 2.2]-2.3) as a distribution-valued process [Wal86, KX95]. However, we find it 
more convenient, as in the case of the white noise generated from a Brownian motion, to work with the stochastic integral with respect to the Brownian sheet $W$, denoted by " $\int \cdot \mathrm{d} W$ " [Wal86, §II], [KX95, Ch. 3]. In our doing so, we bear in mind the formal relationship

$$
\int_{0}^{\infty} \int_{D} f(x, t) \partial_{x t} W(x, t) \mathrm{d} x \mathrm{~d} t=\int_{0}^{\infty} \int_{D} f(x, t) \mathrm{d} W(x, t)
$$

that will inspire the weak formulation 2.10 and the definitions in $\$ 3$. In the particular case where $f$ is the characteristic function of a Borel-measurable set $A \in \mathscr{B}\left(\mathbb{R}^{+} \times D\right)$ of Lebesgue measure $|A|<\infty$ the following basic property of the stochastic integral is satisfied:

$$
\int_{A} \mathrm{~d} W(x, t)=W(A) \in \mathrm{N}(0,|A|),
$$

i.e., $W(A)$ is a Gaussian random variable with mean zero and variance $\left.|A|\right|^{1}$

Since we are interested in numerical solutions, we consider the time domain to be a bounded interval $I=[0, T]$, for some fixed $T \in \mathbb{R}^{+}$. A fundamental property of the stochastic integral is the following well-known $\mathrm{L}_{2}$-isometry, which holds for the Itô integral:

$$
\mathbb{E}\left[\left(\int_{I} \int_{D} f(x, t) \mathrm{d} W(x, t)\right)^{2}\right]=\mathbb{E}\left[\int_{I} \int_{D} f(x, t)^{2} \mathrm{~d} x \mathrm{~d} t\right],
$$

for any $\mathscr{F}_{t}^{W}$-measurable $f \in \mathrm{L}_{2}(I \times D \times \Omega)$, where

$$
\mathscr{F}_{t}^{W}=\sigma\{W(A): A \in \mathscr{B}(I \times D)\}
$$

is the sigma-field (or sigma-algebra) generated by $W$ up to time $t$, and $\mathbb{E}$ denotes the expectation with respect to $(\Omega, \mathscr{F}, P) 2^{2}$

A useful consequence of 2.6 is that

$$
\mathbb{E}\left[\int_{I} \int_{D} f(x, t) \mathrm{d} W(x, t) \int_{I} \int_{D} g(y, s) \mathrm{d} W(y, s)\right]=\mathbb{E}\left[\int_{I} \int_{D} f(x, t) g(x, t) \mathrm{d} x \mathrm{~d} t\right]
$$

for any $\mathscr{F}_{t}{ }^{W}$-measurable $f, g \in \mathrm{L}_{2}(I \times D \times \Omega)$. In the special case where $f$ and $g$ are, respectively, the characteristic functions of two Borel sets $A, B \in \mathscr{B}(I \times D)$ with $|A|,|B|<\infty$, 2.8 implies

$$
\operatorname{Cov}(W(A), W(B))=|A \cap B| .
$$

\subsection{Integral solutions}

By multiplying (1.1) with a test function $\phi \in \mathrm{C}_{\mathrm{c}}^{2}(D \times(0, \infty))$ and using the formal relation (2.4), one can write the problem in the usual weak form ${ }^{3}$

$$
\int_{0}^{\infty} \int_{D}\left(u \partial_{t} \phi-\partial_{x} u \partial_{x} \phi-f_{\epsilon}(u) \phi\right)+\epsilon^{\gamma} \int_{0}^{\infty} \int_{D} \phi \mathrm{d} W=0 .
$$

\footnotetext{
1 For $\mu \in \mathbb{R}$ and $\sigma \in \mathbb{R}^{+}$we denote by $\mathrm{N}\left(\mu, \sigma^{2}\right)$ the class of normally distributed (or Gaussian) random variables of mean $\mu$ and variance $\sigma^{2}$ on the space $\Omega$.

2 In compliance with the standard practice in stochastic differential equations, we write explicitly the probability variable $\omega \in \Omega$ as an argument to random variables only when necessary in order to avoid confusion.

3 Whenever the meaning is clear from the context, for sake of conciseness, we often drop the variables " $x, t$ " and, in nonstochastic integrals, also the corresponding elementary terms " $d$ ".
} 
Despite the above formulation being quite useful, especially for studying a numerical scheme, it is not very convenient to nail down the concept of solution. A rather more convenient way to give rigorous meaning to (1.1) is to look for an integral solution of an equivalent integral equation [DPZ92, Doe87, FJL82, Wal86], as we briefly illustrate next.

Introduce first the corresponding boundary value problem for the stochastic linear heat equation [DPZ92, Wal86]

$$
\begin{gathered}
\partial_{t} Z-\partial_{x x} Z=\partial_{x t} W \\
Z(x, 0)=0 \quad \text { in } D \times \mathbb{R}_{0}^{+}, \\
\partial_{x} Z(1, t)=\partial_{x} Z(-1, t)=0, \quad \forall t \in[0, \infty) .
\end{gathered}
$$

The solution to this problem can be defined as the Gaussian process in space-time produced by the stochastic integral

$$
Z_{t}(x)=Z(x, t):=\int_{0}^{t} \int_{D} G_{t-s}(x, y) \mathrm{d} W(y, s),
$$

where $G$ is the heat kernel for the corresponding homogeneous Neumann problem. In our onedimensional particular case, $G$ can be explicitly written as

$$
G_{t}(x, y)=4 \sum_{k=0}^{\infty}\left(2-\delta_{0}^{k}\right) \cos \frac{\pi k(x+1)}{2} \cos \frac{\pi k(y+1)}{2} \exp \frac{-\pi^{2} k^{2} t}{4},
$$

where $\delta_{0}^{k}$ is the Kronecker symbol.

The integral solution of (1.1) can then be defined as a solution of the equivalent integral equation

$$
u(x, t)=-\int_{0}^{t} \int_{D} G_{t-s}(x, y) f_{\epsilon}(u(y, s)) \mathrm{d} y \mathrm{~d} s+\int_{D} G_{t}(x, y) u_{0}(y) \mathrm{d} y+\epsilon^{\gamma} Z_{t}(x) .
$$

It is known that such a solution exists uniquely as a $\mathrm{C}^{0}(D)$-valued continuous process, $t \mapsto$ $u(\cdot, t)$, adapted to $Z_{t}$, provided the initial condition $u_{0}$ satisfies the Neumann boundary conditions [BDMP95, Wal86, FJL82]. In this article we use this concept of solution which we refer to simply as the solution of Problem (1.1), 2.2)-2.3, and we will denote it by $u$. Notice that $u$ is also referred to by some authors as the Ginzburg-Landau process [BDMP95].

For the aims set in this paper, namely, in order to study the error of convergence of an approximation of the solution of [1.1, we will need a uniform bound for $u$. While in the deterministic case such a bound is a direct consequence of the maximum principle, in the stochastic case one cannot expect to have a uniform bound in the whole probability space. However, a bound on a set with large probability controlled by $\epsilon$ will suffice for our needs. We present an extension of a previously known result of Brassesco et al. [BDMP95, Prop. 5.2].

2.4 Lemma (Probabilistic maximum principle) Let $\gamma>-1 / 2$. For each $T>0$ and $K_{0}>0$ there exist $c_{1}, c_{2}, \delta_{0}>0$ such that if $\left\|u_{0}\right\|_{\mathrm{L}_{\infty}(D)} \leqslant 1+\delta_{0}$ then

$$
P\left\{\sup _{t \in[0, T]}\|u(t)\|_{\mathrm{L}_{\infty}(D)}>1+K_{0}\right\} \leqslant c_{1} \exp \left(-c_{2} / \epsilon^{1+2 \gamma}\right) .
$$

Proof. We reduce the proof to that of [BDMP95, Prop. 5.2] by introducing the time-space rescaling $t \mapsto t / \epsilon^{2}$ and $x \mapsto x / \sqrt{2} \epsilon$ and extending the solution periodically to the whole space so as to 
obtain the proper barrier function. Since we are dealing with the more general case $\gamma>-1 / 2$, while they deal with the case $\gamma=0$ only, we retrace the salient points of their proof. The barrier function $v$ satisfies the following equation—corresponding to [BDMP95, (5.12)]:

$$
\partial_{t} v-\frac{1}{2} \partial_{x x} v+2 v=-3 v^{2}-v^{3}+2^{-1 / 4} \epsilon^{\gamma+1 / 2} \partial_{x t} W .
$$

Consider now the function

$$
V(x, t)=\int_{0}^{t} \exp (-2(t-s)) H_{t-s}^{(\epsilon \sqrt{2})}(x, y) \mathrm{d} W(y, s),
$$

where $H_{t-s}^{(\epsilon \sqrt{2})}$ is the Green operator defined by

$$
\exp (-2 t) H_{t-s}^{(\epsilon \sqrt{2})}=\left(\partial_{t}-\frac{1}{2} \partial_{x x}+2 \text { Id }\right)^{-1},
$$

with homogeneous boundary conditions on $(-1 / \sqrt{2} \epsilon, 1 / \sqrt{2} \epsilon)$. By using [BDMP95] equation (5.2)] with $\lambda=\exp (-(\gamma+1 / 2))$ and adapting properly the proof of [BDMP95] Lemma 2.1] we can easily conclude that for each $b>0$ there exist $c_{1}, c_{2}>0$ such that

$$
P^{\epsilon}\left\{\sup _{t \leqslant T \epsilon^{-2}, x \in \mathbb{R}}\left|\epsilon^{\gamma+1 / 2} V(x, t)\right|>b\right\} \leqslant c_{1} \exp \left(-c_{2} / \epsilon^{1+2 \gamma}\right) .
$$

The rest of the proof is now standard.

\section{White noise approximation}

In order to introduce a finite element method (FEM) that approximates a solution of 2.14, we first need to obtain a weak formulation in the standard sense of PDE and FEM. This is not possible with the presence of the white noise, so we regularize first the problem by replacing the white noise with a smoother stochastic term. Our technique is inspired by that of Allen, Novosel \& Zhang [ANZ98] for the linear heat equation.

\subsection{A piecewise constant approximation of the white noise}

Consider a tensor-product partition of the space-time domain, $\mathscr{D}_{\sigma} \times \mathscr{I}_{\rho}$, where $\sigma, \rho \in \mathbb{R}^{+}$and

$$
\begin{aligned}
& \mathscr{D}_{\sigma}:=\left\{D_{m}: D_{m}:=\left(x_{m-1}, x_{m}\right), m \in[1: M]\right\}, \\
& \mathscr{I}_{\rho}:=\left\{I_{n}: I_{n}:=\left[t_{n-1}, t_{n}\right), n \in[1: N]\right\},
\end{aligned}
$$

are, respectively, a space-domain partition and a time-domain partition; each of these partitions is uniform, that is,

$$
x_{m}-x_{m-1}=\sigma, \quad \forall m \in[1: M], \quad \text { and } \quad t_{n}-t_{n-1}=\rho, \quad \forall n \in[1: N],
$$

and $x_{0}=-1, x_{M}=1, t_{0}=0$ and $t_{N}=T$. We denote by $\chi_{m}=\mathbf{1}_{D_{m}}$ and $\varphi_{n}=\mathbf{1}_{I_{n}}$ the characteristic functions of the space subdomains and time subdomains respectively. 
The (piecewise constant) approximation of white noise, abbreviated by AWN below, is given by the random space-time function

$$
\partial_{x t} \bar{W}(x, t)=\sum_{n=1}^{N} \sum_{m=1}^{N} \bar{\eta}_{m, n} \chi_{m}(x) \varphi_{n}(t)
$$

where the coefficients are the random variables defined by

$$
\bar{\eta}_{m, n}:=\frac{1}{\sigma \rho} \int_{I} \int_{D} \chi_{m}(x) \varphi_{n}(t) \mathrm{d} W(x, t) .
$$

In the following we will use the shorthand

$$
\int_{0}^{t} \int_{D} f(x, s) \mathrm{d} \bar{W}(x, s)=\int_{0}^{t} \int_{D} f(x, s) \partial_{x t} \bar{W}(x, s) \mathrm{d} x \mathrm{~d} s,
$$

in spite of the integral being taken in the classical, nonstochastic sense.

3.2 LEMMA (Moments and independence of the AWN coefficients) The coefficients $\bar{\eta}_{m, n}$ defined in (3.4) are i.i.d. $\mathrm{N}(0,1 / \sigma \rho)$ variables.

Proof. From the definitions of $\bar{\eta}_{m, n}$ and property 2.5 we have

$$
\begin{aligned}
\bar{\eta}_{m, n} & =\frac{1}{\sigma \rho} \int_{I} \int_{D} \chi_{m}(x) \varphi_{n}(t) \mathrm{d} W(x, t) \\
& =\frac{1}{\sigma \rho} \int_{I_{n}} \int_{D_{m}} \mathrm{~d} W(x, t)=\frac{W\left(I_{n} \times D_{m}\right)}{\sigma \rho} \\
& \in \mathrm{N}\left(0, \frac{\left|I_{n} \times D_{m}\right|}{\sigma^{2} \rho^{2}}\right)=\mathrm{N}\left(0, \frac{1}{\sigma \rho}\right) .
\end{aligned}
$$

To show independence compute the covariances for $m, m^{\prime} \in[1: M]$ and $n, n^{\prime} \in[1: N]$, using 2.8, as follows:

$$
\begin{aligned}
(\sigma \rho)^{2} \mathbb{E}\left[\bar{\eta}_{m, n} \bar{\eta}_{m^{\prime}, n^{\prime}}\right] & =\mathbb{E}\left[\int_{I} \int_{D} \chi_{m} \varphi_{n} \mathrm{~d} W \int_{I} \int_{D} \chi_{m^{\prime}} \varphi_{n^{\prime}} \mathrm{d} W\right] \\
& =\int_{I} \int_{D} \chi_{m}(x) \chi_{m^{\prime}}(x) \varphi_{n}(t) \varphi_{n^{\prime}}(t) \mathrm{d} x \mathrm{~d} t \\
& =\delta_{m^{\prime}}^{m} \delta_{n^{\prime}}^{n} \sigma \rho,
\end{aligned}
$$

where $\delta_{j}^{i}$ is the Kronecker symbol.

The AWN has two important technical properties that we state and prove next.

3.3 LEMMA (Approximate Itô-type inequality) For all deterministic functions $f \in \mathrm{L}_{2}(I \times D)$ the following holds true:

$$
\mathbb{E}\left[\left(\int_{I} \int_{D} f(x, t) \mathrm{d} \bar{W}(x, t)\right)^{2}\right] \leqslant \int_{I} \int_{D} f(x, t)^{2} \mathrm{~d} x \mathrm{~d} t .
$$


Proof. Lemma 3.2 and some manipulations yield

$$
\begin{aligned}
\mathbb{E} & {\left[\left(\int_{I} \int_{D} f(x, t) \mathrm{d} \bar{W}(x, t)\right)^{2}\right]=\mathbb{E}\left[\left(\int_{I} \int_{D} f(x, t) \sum_{m, n} \bar{\eta}_{m, n} \chi_{m}(x) \phi_{n}(t) \mathrm{d} x \mathrm{~d} t\right)^{2}\right] } \\
& =\mathbb{E}\left[\left(\sum_{m, n} \bar{\eta}_{m, n} \int_{I_{n}} \int_{D_{m}} f(x, t) \mathrm{d} x \mathrm{~d} t\right)^{2}\right] \\
& =\mathbb{E}\left[\sum_{n m} \bar{\eta}_{m, n}^{2}\left(\int_{I_{n}} \int_{D_{m}} f\right)^{2}+2 \sum_{n \neq n^{\prime}, m \neq m^{\prime}} \bar{\eta}_{m, n} \bar{\eta}_{m^{\prime} n}^{\prime}\left(\int_{I_{n}} \int_{D_{m}} f\right)\left(\int_{I_{n}^{\prime}} \int_{D_{m}^{\prime}} f\right)\right] \\
& =\sum_{m, n} \mathbb{E}\left[\bar{\eta}_{m, n}^{2}\right]\left(\int_{I_{n}} \int_{D_{m}} f\right)^{2}=\sum_{m, n} \frac{1}{\rho \sigma}\left(\int_{I_{n}} \int_{D_{m}} f\right)^{2} \\
& \leqslant \sum_{m, n} \int_{I_{n}} \int_{D_{m}} f^{2}=\int_{I} \int_{D} f(x, t)^{2} \mathrm{~d} x \mathrm{~d} t .
\end{aligned}
$$

In the next-to-last step we use the Cauchy-Schwarz inequality.

3.4 REMARK Lemma 3.3 and 2.6) imply that

$$
\mathbb{E}\left[\left(\int_{I} \int_{D} f(x, t) \mathrm{d} \bar{W}(x, t)\right)^{2}\right] \leqslant \mathbb{E}\left[\left(\int_{I} \int_{D} f(x, t) \mathrm{d} W(x, t)\right)^{2}\right] .
$$

In other words, the $\mathrm{L}_{2}$-type regularity properties of the AWN will be, at the worse, the same as those of the white noise itself.

Since we will need bounds on space-time norms of the AWN, but in probability rather than in expectation, we establish the following basic result.

3.5 LEMMA $\left(\mathrm{L}_{\infty}\left(\mathrm{L}_{2}\right)\right.$ and $\mathrm{L}_{2}\left(\mathrm{~L}_{2}\right)$ bounds for the AWN) For each $K>0$ we have

$$
P\left\{\sup _{t \in[0, T]}\left\|\partial_{x t} \bar{W}(t)\right\|_{\mathrm{L}_{2}(D)} \leqslant K\right\} \geqslant\left[1-\frac{T}{\rho}\left(1+\frac{K^{2}}{2} \rho\right)^{1 / \sigma-1} \exp \left(-\frac{K^{2}}{2} \rho\right)\right]^{+}
$$

and

$$
P\left\{\left\|\partial_{x t} \bar{W}\right\|_{\mathrm{L}_{2}(D \times[0, T])} \leqslant K\right\} \geqslant 1-\left(1+\frac{K^{2}}{2}\right)^{T /(\sigma \rho)-1} \exp \left(-\frac{K^{2}}{2}\right) .
$$

Proof. We proceed in several steps.

Step 1. Recall that $M=2 / \sigma$ and $N=T / \rho$. By the definition of $\partial_{x t} \bar{W}$ we have, for each $t \in[0, T]$ and $n \in[1: N]$ such that $t \in I_{n}$,

$$
\left\|\partial_{x t} \bar{W}(t)\right\|_{\mathrm{L}_{2}(D)}^{2}=\sigma \sum_{m=1}^{M} \bar{\eta}_{m, n}^{2}=\frac{1}{\rho} \sum_{m=1}^{M} \eta_{m, n}^{2},
$$

where the $\eta_{m, n} \in \mathrm{N}(0,1)$. In order to conclude, we will obtain a condition on the right-hand side that makes it smaller than $K^{2}$, for all $n \in[1: N]$. 
Step 2. For each $n \in[1: N]$ we consider the random variable

$$
H_{n}:=\sum_{m=1}^{M} \eta_{m, n}^{2}
$$

Notice that, in view of Lemma 3.2 for $n \neq n^{\prime}, H_{n}$ and $H_{n^{\prime}}$ are independent. Let us fix $n$ for a while and find an event for which $H_{n} \leqslant \rho K^{2}$. By Lemma 3.2 and a basic probability fact [Bil95, Pbm. 20.16], the random variable $H_{n}$ has a chi-squared distribution with $M$ degrees of freedom. Its density is given by

$$
\frac{z^{M / 2-1} \exp (-z / 2)}{2^{M / 2} \Gamma(M / 2)} \quad \text { for } z>0
$$

and 0 for $z \leqslant 0$, where $\Gamma$ is the Euler Gamma-function. Thus we have

$$
P\left\{H_{n} \leqslant \rho K^{2}\right\}=\frac{1}{2^{M / 2} \Gamma(M / 2)} \int_{0}^{\rho K^{2}} z^{M / 2-1} \exp (-z / 2) \mathrm{d} z
$$

Step 3. We next prove a lower bound on this integral in the case where $M$ is even, the odd case being similar. Let $y$ play the role of $\rho K^{2}$ and consider for each $k \in \mathbb{N}_{0}$ the integral

$$
I_{k}:=\int_{0}^{y} z^{k} \exp (-z / 2) \mathrm{d} z
$$

An integration by parts yields the recursive expression

$$
I_{k}=2 k I_{k-1}-2 y^{k} \exp (-y / 2),
$$

which allows, by an inductive argument, to see that

$$
I_{k}=2^{k+1} k !-2 \sum_{i=0}^{k} \frac{k !}{(k-i) !} y^{k-i} 2^{i} \exp (-y / 2) .
$$

An easy manipulation with the binomial formula implies that

$$
I_{k} \geqslant 2^{k+1} k !\left(1-(1+y / 2)^{k} \exp (-y / 2)\right) .
$$

Taking $k=M / 2-1$ in the above and recalling the definition of $I_{k}$ and (3.14) it follows that

$$
P\left\{H_{n} \leqslant \rho K^{2}\right\} \geqslant 1-\left(1+\frac{\rho K^{2}}{2}\right)^{M / 2-1} \exp \left(-\frac{\rho K^{2}}{2}\right),
$$

which implies

$$
P\left\{H_{n} \leqslant \rho K^{2}\right\} \geqslant\left[1-\left(1+\frac{\rho K^{2}}{2}\right)^{M / 2-1} \exp \left(-\frac{\rho K^{2}}{2}\right)\right]^{+}
$$


Step 4. To conclude the proof, we introduce the event

$$
\Omega_{K}^{2}=\bigcap_{n=1}^{N}\left\{H_{n} \leqslant \rho K^{2}\right\}
$$

and we observe that, in view of 3.12, on $\Omega_{K}^{2}$ we have

$$
\left\|\partial_{x t} \bar{W}(t)\right\|_{\mathrm{L}_{2}(D)} \leqslant K, \quad \forall t \in[0, T] .
$$

On the other hand, using the independence of $H_{n}, n \in[1: N]$, the simple fact that $(1-\xi)^{N} \geqslant 1-N \xi$ for $\xi \leqslant 1$ and 3.21 we can estimate the probability

$$
\begin{aligned}
P\left(\Omega_{K}^{2}\right) & =\prod_{n=1}^{N} P\left\{H_{n} \leqslant \rho K^{2}\right\} \\
& \geqslant\left(\left[1-\left(1+\frac{\rho K^{2}}{2}\right)^{M / 2-1} \exp \left(-\frac{\rho K^{2}}{2}\right)\right]^{+}\right)^{N} \\
& \geqslant\left[1-N\left(1+\frac{\rho K^{2}}{2}\right)^{M / 2-1} \exp \left(-\frac{\rho K^{2}}{2}\right)\right]^{+} .
\end{aligned}
$$

By replacing $N=T / \rho$ and $M=2 / \sigma$ we get 3.10 .

Step 5. Estimate 3.11 is obtained simply by using $\left(3.21\right.$ with $\rho K^{2}$ and $M$ replaced by $K^{2}$ and $M N$ respectively.

3.6 REMARK (Alternative proof) As pointed out by one of the referees, it is possible to prove Lemma 3.5 more directly, by using martingale inequalities.

3.7 REMARK (Interpretation of (3.10) and 3.11) We may rewrite the term appearing in 3.10) as

$$
\frac{T}{\rho}\left(1+\frac{K^{2}}{2} \rho\right)^{1 / \sigma-1} \exp \left(-\frac{K^{2}}{2} \rho\right)=: T \exp F(\rho, \sigma, K) .
$$

A practical way to use such a result is by fixing first $T, \rho, \sigma$ in $\mathbb{R}^{+}$and then requiring $K$ to be big enough such that $T \exp F(\rho, \sigma, K) \ll 0$. This is made possible by the fact that $\lim _{K \rightarrow \infty} F(\rho, \sigma, K)=-\infty$ for any fixed $\rho, \sigma \in \mathbb{R}^{+}$. The same type of observation is also valid for 3.11.

\section{The regularized solution}

We now introduce the regularized solution to problem (1.1), 2.2 - 2.3, which we obtain by replacing the white noise by the AWN in 1.1. The role of the regularized problem is pivotal in devising a numerical scheme to approximate the stochastic Allen-Cahn problem. We discuss the approximation properties of this regularization with respect to the original problem.

4.1 Definition The regularized solution, $\bar{u}$, of the noisy Allen-Cahn problem is the unique continuous solution of the integral equation

$$
\begin{aligned}
\bar{u}(x, t)= & -\int_{0}^{t} \int_{D} G_{t-s}(x, y) f_{\epsilon}(\bar{u}(y, s)) \mathrm{d} y \mathrm{~d} s \\
& +\int_{D} G_{t}(x, y) u_{0}(y) \mathrm{d} y+\epsilon^{\gamma} \int_{0}^{t} \int_{D} G_{t-s}(x, y) \mathrm{d} \bar{W}(y, s) .
\end{aligned}
$$


4.2 LEMMA (Maximum principle for regularized solutions) For fixed $T, K_{0}>0$, there exist $\delta_{0}, c_{1}, c_{2}>0$, independent of $\epsilon$, such that if $\left\|u_{0}\right\|_{\mathrm{L}_{\infty}(D)} \leqslant 1+\delta_{0}$ then

$$
P\left\{\sup _{t \in[0, T]}\|\bar{u}(t)\|_{\mathrm{L}_{\infty}(D)} \leqslant 1+K_{0}\right\} \geqslant 1-c_{1} \exp \left(-c_{2} / \epsilon^{1+2 \gamma}\right) .
$$

Proof. We follow exactly the proof of Lemma 2.4. by observing that 3.9 ensures that all the estimates for the stochastic integrals of the white noise can be "translated" into corresponding estimates for the integrals of the approximate white noise. The constants appearing in this theorem can therefore be taken to be the same that appear in $\$ 2.4$

4.3 REMARK (Regularized solution is strong solution) Notice that the regularized solution $\bar{u}$ of (4.1) is in fact a weak solution in the PDE sense, i.e., $\bar{u}(t ; \omega) \in \mathrm{H}^{1}(D)$ and $\partial_{t} \bar{u}(t ; \omega) \in \mathrm{L}_{2}(D)$ for all $t \in(0, T]$ and $\omega \in \Omega$, and the following weak formulation is satisfied:

$$
\begin{aligned}
\left\langle\partial_{t} \bar{u}(t ; \omega), \phi\right\rangle+\left\langle\partial_{x} \bar{u}(t ; \omega), \partial_{x} \phi\right\rangle+ & \left\langle f_{\epsilon}(\bar{u}(t ; \omega)), \phi\right\rangle \\
& =\epsilon^{\gamma}\left\langle\partial_{x t} \bar{W}(t ; \omega), \phi\right\rangle, \quad \forall \phi \in \mathrm{H}_{0}^{1}(D), t \in(0, T], \\
\bar{u}(0 ; \omega)=u_{0}, &
\end{aligned}
$$

for each $\omega \in \Omega$ (the notation $\langle\cdot, \cdot\rangle$ indicating the inner product in $\mathrm{L}_{2}(D)$ ). Indeed, each of the AWN's realizations, $\partial_{x t} \bar{W}(\omega)$, is a piecewise constant space-time function. For each such realization the usual regularity theory for semilinear parabolic equations with piecewise continuous data can be applied and the corresponding weak formulation written down [LSU68].

Our next goal is to show that the regularized approximate solution converges to the solution $u$. For this we will estimate the regularization error

$$
e(x, t)=u(x, t)-\bar{u}(x, t),
$$

in terms of the white noise regularization parameters $\sigma$ and $\rho$, and show that it converges to zero in an appropriate sense.

4.4 THEOREM (Convergence to the stochastic solution) For a fixed $T$, there exist constants $c_{1}, c_{2}$, $C_{1}$ and $C_{2}$ such that to each $\epsilon \in(0,1)$ there correspond an event $\Omega_{\epsilon}^{\infty}$ and a constant $C_{\epsilon}>0$ such that

$$
\begin{gathered}
P\left(\Omega_{\epsilon}^{\infty}\right) \geqslant 1-2 c_{1} \exp \left(-c_{2} / \epsilon^{1+2 \gamma}\right), \\
\int_{\Omega_{\epsilon}^{\infty}}\left(\int_{0}^{T} \int_{D}|\bar{u}-u|^{2}\right) \mathrm{d} P \leqslant C_{\epsilon}\left(C_{1} \rho^{1 / 2}+C_{2} \frac{\sigma^{2}}{\rho^{1 / 2}}\right), \quad \forall \sigma, \rho>0 .
\end{gathered}
$$

Proof. We proceed by steps.

Step 1. By the integral representations of $u$, 2.14), and $\bar{u}$, (4.1), we can also represent the error as an integral

$$
\begin{aligned}
e(x, t)= & \int_{0}^{t} \int_{D} G_{t-s}(x, y)\left(f_{\epsilon}(\bar{u}(y, s))-f_{\epsilon}(u(y, s))\right) \mathrm{d} y \mathrm{~d} s \\
& +\epsilon^{\gamma} \int_{0}^{t} \int_{D} G_{t-s}(x, y)(\mathrm{d} W(y, s)-\mathrm{d} \bar{W}(y, s))
\end{aligned}
$$

for all $(x, t) \in D \times(0, T]$. So our task is now to bound the terms on the right-hand side of 4.7) in the appropriate norm. 
Step 2. In view of the maximum principle for both the exact solution, $\$ 2.4$ and the approximate solution, $\$ 4.2$ there exists an event $\Omega_{\epsilon}^{\infty} \subset \Omega$ such that

$$
P\left(\Omega_{\epsilon}^{\infty}\right) \geqslant 1-2 c_{1} \exp \left(-c_{2} / \epsilon^{1+2 \gamma}\right)
$$

and

$$
\Omega_{\epsilon}^{\infty} \subset\left\{\|u(t)\|_{\mathrm{L}_{\infty}(D)},\|\bar{u}(t)\|_{\mathrm{L}_{\infty}(D)} \leqslant 3, \forall t \in[0, T]\right\} .
$$

The choice of the number 3 is quite arbitrary here. In fact any number of the form $1+K_{0}$ with $K_{0}>0$ will do, with the appropriate change of constants. This and the local Lipschitz continuity of $f$ imply that

$$
\left|f_{\epsilon}(\bar{u})-f_{\epsilon}(u)\right| \leqslant \frac{28}{\epsilon^{2}}|\bar{u}-u| \quad \text { on } \Omega_{\epsilon}^{\infty} .
$$

Step 3. Working now on the event $\Omega_{\epsilon}^{\infty}$ and introducing the functions

$$
\begin{aligned}
\varepsilon(r) & :=\int_{0}^{r} \int_{D} e(x, t)^{2} \mathrm{~d} x \mathrm{~d} t, \\
\phi(r) & :=\int_{0}^{r} \int_{D}\left|\int_{0}^{t} \int_{D} G_{t-s}(x, y)(\mathrm{d} W(y, s)-\mathrm{d} \bar{W}(y, s))\right|^{2} \mathrm{~d} x \mathrm{~d} t
\end{aligned}
$$

for all $r \in[0, T]$, we infer from (4.7) that

$$
\varepsilon(r) \leqslant 2 \int_{0}^{r} \int_{D}\left(\int_{0}^{t} \int_{D}\left|G_{t-s}(x, y)\right| \frac{28}{\epsilon^{2}} e(y, s) \mathrm{d} y \mathrm{~d} s\right)^{2} \mathrm{~d} x \mathrm{~d} t+2 \epsilon^{2 \gamma} \phi(r) .
$$

The integral in (4.13) can be bounded, using the Cauchy-Schwarz inequality, by

$$
2 \frac{28^{2}}{\epsilon^{4}} \int_{0}^{r} \int_{D}\left(\int_{0}^{t} \int_{D}\left|G_{t-s}(x, y)\right|^{2} \mathrm{~d} y \mathrm{~d} s \int_{0}^{t} \int_{D} e(y, s)^{2} \mathrm{~d} y \mathrm{~d} s\right) \mathrm{d} x \mathrm{~d} t=\int_{0}^{r} z(t) \varepsilon(t) \mathrm{d} t
$$

where

$$
z(t):=2 \frac{28^{2}}{\epsilon^{4}} \int_{D} \int_{0}^{t} \int_{D}\left|G_{t-s}(x, y)\right|^{2} \mathrm{~d} y \mathrm{~d} s \mathrm{~d} x .
$$

Inequality 4.13 implies

$$
\varepsilon(r) \leqslant \phi(r)+\int_{0}^{r} z(t) \varepsilon(t) \mathrm{d} t
$$

for each $r \in I$. Applying the Gronwall lemma to this inequality we obtain

$$
\varepsilon(T) \leqslant \exp \left(\int_{0}^{T} z(t) \mathrm{d} t\right) \epsilon^{2 \gamma} \phi(T) \leqslant C_{\epsilon} \phi(T),
$$

where—by estimating the heat kernel—the constant is given by

$$
C_{\epsilon}:=\epsilon^{2 \gamma} \exp \left(\frac{28^{2} T}{12 \epsilon^{4}}\right) .
$$


Step 4. By summing with respect to $P$ on the event $\Omega_{\epsilon}^{\infty}$ both members of this inequality we obtain

$$
\int_{\Omega_{\epsilon}^{\infty}} \int_{0}^{T} \int_{D}|\bar{u}-u|^{2} \mathrm{~d} x \mathrm{~d} t \mathrm{~d} P \leqslant C_{\epsilon} \int_{\Omega_{\epsilon}^{\infty}} \phi(T) \mathrm{d} P \leqslant C_{\epsilon} \mathbb{E}[\phi(T)] .
$$

We conclude by observing [ANZ98, Lem. 2.3] that there exist $C_{1}, C_{2}>0$, depending only on $T$, such that

$$
\mathbb{E}[\phi(T)] \leqslant C_{1} \rho^{1 / 2}+C_{2} \frac{\sigma^{2}}{\rho^{1 / 2}} .
$$

Thus we established that

$$
\int_{\Omega_{\epsilon}^{\infty}} \int_{0}^{T} \int_{D}|\bar{u}-u|^{2} \mathrm{~d} x \mathrm{~d} t \mathrm{~d} P \leqslant C_{\epsilon}\left(C_{1} \rho^{1 / 2}+C_{2} \frac{\sigma^{2}}{\rho^{1 / 2}}\right),
$$

as claimed.

4.5 REMARK (about the constant $C_{\epsilon}$ ) Theorem 4.4 ensures that, for fixed $T$ and $\epsilon$, the approximate solution $\bar{u}$ converges to $u$ as $\rho, \sigma \rightarrow 0$. The constant $C_{\epsilon}$ appearing in the estimate depends exponentially on both $1 / \epsilon^{4}$ and $T$, thus for small $\epsilon$, or large $T$, this might force us to take very small $\rho$ and $\sigma$. This fact should be taken into account in practice. The bound we have proved seems to be pessimistic though, as the choice of $\sigma$ and $\rho$, used in our subsequent numerical experiments, indicates.

4.6 REMARK (Convergence rate) Recalling that $\rho$ and $\sigma$ can be thought of as discretization (in addition to regularization) parameters, the convergence rate found in (4.6) is in accordance with standard results for linear parabolic equations, e.g. in [ANZ98]. Note that for $\rho=C \sigma^{2}$, the socalled "parabolic space-time scaling", we obtain the right balance between the two terms on the right-hand side of 4.6 .

\section{The regularized solution's limit for $\epsilon \rightarrow 0$}

In this section we focus on the relation between the regularized stochastic Allen-Cahn problem (4.3) and the deterministic version. The reason to do this is to find, in an analytical setting, what conditions should be imposed on the regularization parameters, $\rho$ and $\sigma$, for the noise to be captured in the regularized equation.

We mainly show that the error between $\bar{u}$ and the deterministic solution to the Allen-Cahn problem, $q$, in an appropriate probability- $\mathrm{L}_{\infty}\left(0, T ; \mathrm{L}_{2}\right)$ sense, is of order $\mathrm{O}\left(\epsilon^{3}\right)$ as $\epsilon \rightarrow 0$ for fixed $\gamma>3$ and $\rho, \sigma>0$. In $\$ 5.6$ we give an interpretation of this result as an evaluation of the risk of obtaining a poor resolution of the noise for fixed $\rho$ and $\sigma$ and too small $\epsilon$. This poor resolution may lead to the disappearance of the stochastic effects in the regularized equation, even after the appropriate rescaling, because $\bar{u}$ becomes much closer to $q$ than $u$, with respect to $\epsilon$. This point is further investigated numerically in $\$ 7$

Our proof makes use of a spectrum estimate result for the linearized Allen-Cahn operator [Che94, dMS95], which is recalled in Theorem 5.2, and the $\mathrm{L}_{2}(D)$ estimate on the noise given by 3.5. The proof's technique is a continuous data dependence result for parabolic equations based on a Bernoulli-Gronwall type argument, in the spirit of Feng \& Wu [FW05].

The result holds for $\gamma>3$ and it is an open problem, as far as we know, to find the critical $\gamma$ for which the result ceases to hold. 


\subsection{Deterministic solution and resolved profiles}

Denote by $q$ the (classical) solution of the problem

$$
\begin{aligned}
\partial_{t} q-\partial_{x x} q+f_{\epsilon}(q) & =0 & & \text { in } D \times I, \\
q(0) & =u_{0} & & \text { on } D, \\
\partial_{x} q(t, 0)=\partial_{x} q(t, 1) & =0, & & t \in I .
\end{aligned}
$$

We also consider the function $q_{0}$ of the space variable only defined as the unique solution to

$$
-q_{0}^{\prime \prime}+f_{1}\left(q_{0}\right)=0 \quad \text { in } \mathbb{R}, \quad q_{0}( \pm \infty)= \pm 1, q_{0}(0)=0 .
$$

(In fact, $q_{0}=$ tanh.) We will assume from now on that $u_{0}$ is a resolved profile solution, which is defined to be an $\epsilon$-linear perturbation of an $\epsilon$-rescaled and shifted $q_{0}$. That is, for all $x \in D$, $u_{0}(x)=q_{0}\left(\left(x-x_{0}\right) / \epsilon\right)+\epsilon p_{0}(x)$ where $x_{0} \in D$, and $p_{0}$ is such that $u_{0}$ satisfies the Neumann boundary conditions $u_{0}^{\prime}( \pm 1)=0$. With this choice of initial condition the linearization of the operator $u \mapsto-\partial_{x x} u+f_{\epsilon}(u)$ about $q$ enjoys the following spectral property.

5.2 TheOREM (Spectrum estimate [Che94, dMS95]) There exists a constant $\lambda_{0}>0$ such that for any $\epsilon \in(0,1]$ we have

$$
\left\|\partial_{x} \phi\right\|_{\mathrm{L}_{2}(D)}^{2}+\left\langle f_{\epsilon}^{\prime}(q) \phi, \phi\right\rangle \geqslant-\lambda_{0}\|\phi\|_{\mathrm{L}_{2}(D)}^{2}, \quad \forall \phi \in \mathrm{H}^{1}(D) .
$$

It is also a well-known consequence of the maximum principle that if $\left|u_{0}\right| \leqslant 1$ (which is the case when $u_{0}$ is a resolved profile) then $|q| \leqslant 1$.

The main result of this section is

5.3 LEMMA (Continuous dependence for the regularized-deterministic error) There is a bounded and nonincreasing function $K_{1}:[0, \infty) \rightarrow \mathbb{R}$ and a constant $K_{2}$, both depending only on $\lambda_{0}$, such that

$$
\|\bar{u}(t)-q(t)\|_{\mathrm{L}_{2}(D)} \leqslant K_{2} \epsilon^{3}
$$

provided

$$
\int_{0}^{t}\left\|\partial_{x t} \bar{W}(s)\right\|_{\mathrm{L}_{2}(D)}^{2} \exp \left(-\left(3+2 \lambda_{0}\right) s\right) \mathrm{d} s \leqslant K_{1}(t) \epsilon^{6-2 \gamma},
$$

for $t \in[0, T]$.

Proof. We divide the proof into several steps and we denote in it $\|\cdot\|_{\mathrm{L}_{2}(D)}$ simply by $\|\cdot\|$.

Step 1. We start by deriving an energy inequality for the error

$$
\bar{e}:=\bar{u}-q .
$$

Since $\bar{u}$ satisfies the weak formulation 4.3 and $q$ is a classical solution, we can write the following PDE in its weak formulation for $\bar{e}$ :

$$
\left\langle\partial_{t} \bar{e}, \phi\right\rangle+\left\langle\partial_{x} \bar{e}, \partial_{x} \phi\right\rangle+\left\langle f_{\epsilon}^{\prime}(q) \bar{e}, \phi\right\rangle=\epsilon^{\gamma}\left\langle\partial_{x t} \bar{W}, \phi\right\rangle-\frac{1}{\epsilon^{2}}\left\langle\bar{r} \bar{e}^{2}, \phi\right\rangle, \quad \forall \phi \in \mathrm{H}^{1}(D),
$$

where

$$
\bar{r}:=3 q+\bar{e}=2 q+\bar{u} .
$$


Testing with $\bar{e}$ in (5.9) we obtain

$$
\left\langle\partial_{t} \bar{e}, \bar{e}\right\rangle+\left\|\partial_{x} \bar{e}\right\|^{2}+\left\langle f_{\epsilon}^{\prime}(q) \bar{e}, \bar{e}\right\rangle \leqslant \epsilon^{\gamma}\left\langle\partial_{x t} \bar{W}, \bar{e}\right\rangle-\frac{1}{\epsilon^{2}}\left\langle\bar{r}, \bar{e}^{3}\right\rangle .
$$

Step 2. The next step is to bound the terms on the right-hand side of 5.11]. The first term can be written as

$$
\epsilon^{\gamma}\left\langle\partial_{x t} \bar{W}(t), \bar{e}(t)\right\rangle \leqslant \frac{\epsilon^{2 \gamma}}{2}\left\|\partial_{x t} \bar{W}(t)\right\|^{2}+\frac{1}{2}\|\bar{e}(t)\|^{2} .
$$

To produce a bound on the second term of the right-hand side of 5.11) we use 5.10, valid in one spatial dimension, to obtain

$$
\left\langle\bar{r}(t), \bar{e}(t)^{3}\right\rangle=3\left\langle q, \bar{e}(t)^{3}\right\rangle+\|\bar{e}(t)\|_{\mathrm{L}_{4}(D)}^{4} .
$$

By the fact that $|q| \leqslant 1$ and the Sobolev embedding $\mathrm{H}^{1}(D) \hookrightarrow \mathrm{L}_{\infty}(D)$, valid for $D \subset \mathbb{R}$, the first term on the right-hand side of (5.13) can be bounded using

$$
\begin{aligned}
\left|3\left\langle q, \bar{e}(t)^{3}\right\rangle\right| & \leqslant 3\|\bar{e}(t)\|_{\mathrm{L}_{\infty}(D)}\|\bar{e}(t)\|^{2} \leqslant C_{1}\|\bar{e}(t)\|_{\mathrm{H}^{1}(D)}\|\bar{e}(t)\|^{2} \\
& \leqslant \lambda_{1} \epsilon^{4}\|\bar{e}(t)\|_{\mathrm{H}^{1}(D)}^{2}+\frac{C_{1}^{2}}{4 \lambda_{1} \epsilon^{4}}\|\bar{e}(t)\|^{4}
\end{aligned}
$$

where $C_{1}$ is 3 times the Sobolev embedding constant for $D$ and $\lambda_{1}:=\min \left\{1, \lambda_{0}\right\}$ (the reason for this choice will be apparent in the next step) with $\lambda_{0}$ from (5.5). As a consequence we have

$$
-\frac{1}{\epsilon^{2}}\left\langle\bar{r}, \bar{e}^{3}\right\rangle \leqslant \lambda_{1} \epsilon^{2}\|\bar{e}\|_{\mathrm{H}^{1}(D)}^{2}+\frac{C_{2}}{\epsilon^{6}}\|\bar{e}\|^{4}-\frac{1}{\epsilon^{2}}\|\bar{e}\|_{\mathrm{L}_{4}(D)}^{4}
$$

where $C_{2}=C_{1}^{2} / 4 \lambda_{1}$.

Step 3. Owing to the spectrum estimate (5.5) and the fact that $f^{\prime}(q) \geqslant-1$ we have

$$
\begin{aligned}
\left\|\partial_{x} \bar{e}\right\|^{2}+\left\langle f_{\epsilon}^{\prime}(q), \bar{e}^{2}\right\rangle & =: A=\left(1-\epsilon^{2}\right) A+\epsilon^{2} A \\
& \geqslant-\left(1-\epsilon^{2}\right) \lambda_{0}\|\bar{e}\|^{2}+\epsilon^{2}\left\|\partial_{x} \epsilon\right\|^{2}-\|\bar{e}\|^{2} \\
& =-\left(\left(1-\epsilon^{2}\right) \lambda_{0}+1\right)\|\bar{e}\|^{2}+\epsilon^{2}\left\|\partial_{x} \bar{e}\right\|^{2} \\
& =-\left(1+\lambda_{0}\right)\|\bar{e}\|^{2}+\epsilon^{2}\left(\left\|\partial_{x} \bar{e}\right\|^{2}+\lambda_{0}\|\bar{e}\|^{2}\right) \\
& \geqslant-\left(1+\lambda_{0}\right)\|\bar{e}\|^{2}+\lambda_{1} \epsilon^{2}\|\bar{e}\|_{\mathrm{H}^{1}(D)}^{2} .
\end{aligned}
$$

The inequalities (5.11), 5.12), (5.16) and (5.15) lead to

$$
\begin{aligned}
\frac{1}{2} \mathrm{~d}_{t}\|\bar{e}(t)\|^{2} & -\left(1+\lambda_{0}\right)\|\bar{e}(t)\|^{2} \\
& \leqslant \frac{\epsilon^{2 \gamma}}{2}\left\|\partial_{x t} \bar{W}(t)\right\|^{2}+\frac{1}{2}\|\bar{e}(t)\|^{2}+\frac{C_{2}}{\epsilon^{6}}\|\bar{e}(t)\|^{4}-\frac{1}{\epsilon^{2}}\|\bar{e}(t)\|_{\mathrm{L}_{4}(D)}^{4}, \quad \forall t \in I .
\end{aligned}
$$

Consider, for the rest of the proof, the following notation:

$$
\begin{aligned}
& g(t):=\|\bar{e}(t)\|^{2}, \quad a:=3+2 \lambda_{0}, \quad b:=2 C_{2} / \epsilon^{6}, \\
& r(t):=\epsilon^{2 \gamma}\left\|\partial_{x t} \bar{W}(t)\right\|^{2}-\|\bar{e}(t)\|_{\mathrm{L}_{4}(D)}^{4} .
\end{aligned}
$$


Then (5.17) implies

$$
g^{\prime}(t) \leqslant a g(t)+b g(t)^{2}+r, \quad \forall t \in[0, T] .
$$

Step 4. To proceed we will apply a Bernoulli differential inequality technique, which generalizes the Gronwall lemma, in order to get a bound on $g(t)$. We follow Feng \& Wu [FW05, Lem. 2.1].

Fix a $t \in[0, T]$ and let

$$
\varrho(s):=\int_{0}^{s} \exp (-a \tau) r(\tau) \mathrm{d} \tau \quad \text { and } \quad p(s):=p_{t}(s)=(\varrho(t)-\varrho(s)) \exp (a s)
$$

for all $s \in[0, t]$. Since

$$
p^{\prime}(s)=\mathrm{d}_{s} p_{t}(s)=-r(s)+a p(s) \quad \text { and } \quad p(s) \geqslant 0 \quad \text { for } s \in[0, t],
$$

we may write

$$
\mathrm{d}_{s}(g(s)+p(s)) \leqslant a(g(s)+p(s))+b(g(s)+p(s))^{2} \quad \text { for } s \in[0, t] .
$$

Introducing $z(s):=1 /(g(s)+p(s))$, we can rewrite this inequality as

$$
z^{\prime}(s)+a z(s) \geqslant-b, \quad \forall s \in[0, t] .
$$

Multiplying by $\exp (a s)$ and integrating over $[0, t]$ we obtain

$$
z(t) \geqslant z(0) \exp (-a t)-\frac{b(1-\exp (-a t))}{a} .
$$

If we note that $g(0)=\|\bar{e}(0)\|=0, p_{t}(0)=\varrho(t)$ and $p_{t}(t)=0$, this yields

$$
\frac{1}{g(t)} \geqslant \frac{a-b \varrho(t)(\exp (a t)-1)}{a \exp (a t) \varrho(t)} \text {. }
$$

We now invert both sides of this inequality, under the sufficient condition that

$$
a-b \varrho(t)(\exp (a t)-1) \geqslant 0
$$

and we get

$$
\|\bar{e}(t)\|^{2} \leqslant \frac{a \exp (a t) \varrho(t)}{a-b \varrho(t)(\exp (a t)-1)} .
$$

Step 5. To conclude we want to interpret this result more explicitly. Let us replace first 5.26 by the sufficient condition

$$
a-b \varrho(t)(\exp (a t)-1) \geqslant \delta(t),
$$

for some $\delta(t)>0$ that will be chosen appropriately. This is equivalent to

$$
\int_{0}^{t} \exp (-a s) r(s) \mathrm{d} s(=\varrho(t)) \leqslant \frac{a-\delta(t)}{b(\exp (a t)-1)} .
$$


This can be ensured if we assume

$$
\int_{0}^{t}\left\|\partial_{x t} \bar{W}(s)\right\|^{2} \exp (-a s) \mathrm{d} s \leqslant \frac{(a-\delta(t)) \epsilon^{6-2 \gamma}}{2 C_{2}(\exp (a t)-1)} .
$$

Under this condition we obtain the bound

$$
\|\bar{e}(t)\|^{2} \leqslant \frac{a(a-\delta(t)) \exp (a t)}{2 C_{2} \delta(t)(\exp (a t)-1)} \epsilon^{6} .
$$

Step 6. We conclude by taking

$$
\delta(t):=\max \{a-\exp (a t)+1, a / 2\},
$$

i.e.,

$$
\delta(t):= \begin{cases}a-\exp (a t)+1 & \text { for } t \leqslant t_{a} \\ a / 2 & \text { for } t>t_{a}\end{cases}
$$

where $t_{a}=\log (1+a / 2) / a$. Then, after putting

$$
K_{1}(t):=\frac{\min \{1, a / 2(\exp (a t)-1)\}}{2 C_{2}} \quad \text { for } t \geqslant 0,
$$

condition 5.29 may be replaced by

$$
\int_{0}^{t}\left\|\partial_{x t} \bar{W}(s)\right\|^{2} \exp (-a s) \mathrm{d} s \leqslant K_{1}(t) \epsilon^{6-2 \gamma},
$$

a condition under which we have, from 5.31,

$$
\|\bar{e}(t)\|^{2} \leqslant K_{2}^{2} \epsilon^{6},
$$

where $K_{2}^{2}:=\sup _{[0, \infty)} K_{1}(t)^{2} \exp (a t)<\infty$.

As a consequence of this estimate we state the following result, which, roughly speaking, implies that in order for the noise to have the chance of persisting in the limit, as $\epsilon \rightarrow 0$, the parameters $\rho, \sigma$ must also go to zero.

5.4 TheOREM (Low-intensity approximate white noise) There exists a constant $C=C\left(\lambda_{0}\right)$ such that for all fixed $\gamma>3, \rho, \sigma, T>0$ we have

$$
\lim _{\epsilon \rightarrow 0} P\left\{\omega \in \Omega: \sup _{[0, T]}\|\bar{u}(\cdot ; \omega)-q\|_{\mathrm{L}_{2}(D)}<C \epsilon^{3}\right\}=1 .
$$

Proof. Let $C=K_{2}$ in 5.6. Choosing to use an $\mathrm{L}_{2}\left(0, t ; \mathrm{L}_{2}(D)\right)$-norm estimate for the white noise, for the estimate (5.6), in view of the monotonicity of $K_{1}$, it is enough to assume the sufficient condition

$$
\int_{0}^{t}\left\|\partial_{x t} \bar{W}(s)\right\|^{2} \mathrm{~d} s \leqslant K_{1}(T) \epsilon^{6-2 \gamma}
$$

According to (3.11), this condition is satisfied with probability

$$
1-\left(1+\frac{K_{1}(T)}{2} \epsilon^{6-2 \gamma}\right)^{T / \sigma \rho-1} \exp \left(-\frac{K_{1}(T)}{2} \epsilon^{6-2 \gamma}\right)
$$


Since $\gamma>3$, we have $6-2 \gamma<0$ and, for fixed $\sigma, \rho$ and $T>0$, it is possible to make this probability arbitrarily close to 1 for $\epsilon>0$ small enough, as claimed.

5.5 REMARK Note that, it is also possible to obtain a variant of 5.4 by employing 3.10 instead of (3.11). This leads to slightly better lower estimates of the probability for the same $\epsilon>0$ for longer time $T$.

\subsection{White noise resolution by the AWN}

We now describe one interpretation of Theorem 5.4 .

Note first that, in the theorem's statement, the AWN regularization parameters $\sigma$ and $\rho$ are kept fixed while $\epsilon \rightarrow 0$. It is well known that, if $u_{0}$ is a resolved profile with center at $x_{0}$ (see $\$ 7.2$ for a definition of center), i.e., $u_{0}=\tanh \left(\left(x-x_{0}\right) / 2 \epsilon\right)$, as $\epsilon \rightarrow 0$, the solution $q=q^{\epsilon}$ of (5.1) converges, in an appropriate sense, to the stationary step function $\chi_{x_{0}}:=\mathbf{1}_{\left(x_{0}, \infty\right)}-\mathbf{1}_{\left(-\infty, x_{0}\right)}$. So Theorem 5.4 is saying that for fixed $\rho, \sigma>0$ and for $\epsilon \rightarrow 0$, the solution, $\bar{u}$, of the regularized problem (4.3) converges to this stationary step function.

Second, we note that, owing to a result by Funaki [Fun95, Theorem 8.1] or a similar one by Brassesco et al. [BDMP95], there exists a stochastic process $(t, \omega) \mapsto \xi_{t}^{\epsilon}(\omega)$ such that

$$
\lim _{\epsilon \searrow 0} P\left\{\omega \in \Omega: \sup _{t \in\left[0, T \epsilon^{-1-2 \gamma}\right]}\left\|u(\cdot, t ; \omega)-\chi_{\xi_{t}^{\epsilon}(\omega)}\right\|_{\mathrm{L}_{2}(D)}>\delta\right\}=0
$$

for each fixed $\delta>0$, where $\chi_{x_{0}}$ is the step function defined above, and $(t, \omega) \mapsto \xi_{t}^{\epsilon}$ is a stochastic process which converges as $\epsilon \rightarrow 0$, in an appropriate sense (in law), to the standard Brownian motion rescaled as to have diffusion coefficient $\sqrt{c_{0}} \epsilon^{1 / 2+\gamma}$ where $c_{0}=3 \sqrt{2} / 4$. Of course, as $\epsilon \rightarrow 0, \xi_{t}^{\epsilon}(\omega) \rightarrow x_{0}$, where $x_{0}$ is the center of the initial condition $u_{0}$; this implies that the limits of $u$ and $\bar{u}$ are consistent, as $\epsilon \rightarrow 0$, even when $\rho$ and $\sigma$ are kept fixed.

Suppose now that one wishes, in view of Theorem 4.4. to use the regularized solution $\bar{u}(t)$, instead of $u(t)$, to approximate the diffusion coefficient of the process $t \mapsto \xi_{t}^{\epsilon}$. One way of doing this would be to approximate (numerically) $\bar{u}(t, \omega)$ for $\omega \in \Omega$ (or a discrete analog), find its center, if it exists, $\bar{\xi}_{t}^{\epsilon}(\omega)$, and finally compute its average (excluding solutions that have no center) and its variance over $\omega \in \Omega$. The resulting variance, rescaled appropriately, i.e., $t \mapsto \operatorname{Var}\left[\xi_{t}^{\epsilon}\right] / \epsilon^{1+2 \gamma}$, is expected to converge to a linear function $t \mapsto c_{0} t$ as $\epsilon \rightarrow 0$. This rescaling, which we shall call the Mueller-Funaki rescaling [Fun95], is necessary in order to get a result that is essentially independent of $\epsilon$ and thus easy to visualize.

Theorem 5.4 tells us that, for fixed $\rho, \sigma>0$, the rate of convergence of $\bar{u} \rightarrow q$ is $\mathrm{O}\left(\epsilon^{3} / 2\right)$. Since the distance $\left(\mathbb{E}\left\|q(t)-\chi \xi_{t}\right\|^{2}\right)^{1 / 2}$, as can be seen using a piecewise constant approximation of tanh, is $\mathrm{O}\left(\epsilon^{1 / 2}\right)$, it follows that $\bar{u}$ is closer to $q$ than $\chi \xi_{t}^{\epsilon}$ and that any statistics conducted on $\bar{u}$ may lead to wrong results. This is a strong indication, which is confirmed by the numerical results in $\$ 7$, that in order to capture the stochastic effects the parameters $\sigma$ and $\rho$ must be chosen as functions of $\epsilon$. Note that a similar conclusion can be derived from Theorem 4.4 in case the dependence of $C_{\epsilon}$ proves to be effective, but the nature of this similar conclusion has its roots in deterministic considerations rather than stochastic ones.

Although we have proved Theorem 5.4 for values of $\gamma>3$, it is natural to expect similar results for lower values of $\gamma$. In fact, our numerical experiments in Section 7 indicate that this is the case. 


\section{An Euler-Galerkin finite element scheme}

We now introduce finite element discretization of the regularized problem 4.3.

\subsection{Discretization partitions}

We begin by introducing the space and time partitions

$$
\begin{aligned}
\mathscr{D}_{h} & :=\left\{D_{m}^{\prime}: D_{m}^{\prime}:=\left(x_{m-1}^{\prime}, x_{m}^{\prime}\right), m \in\left[1: M^{\prime}\right]\right\}, \\
\mathscr{I}_{k} & :=\left\{I_{n}^{\prime}: I_{n}^{\prime}:=\left[t_{n-1}^{\prime}, t_{n}^{\prime}\right), n \in\left[1: N^{\prime}\right]\right\} .
\end{aligned}
$$

These partitions do not necessarily coincide with the partitions $\mathscr{D}_{\sigma}$ and $\mathscr{I}_{\rho}$ used for the regularization procedure in $\$ 3.1$. Bearing in mind that this setting could be further generalized, we limit ourselves here to the case where the numerical discretization partitions, $\mathscr{D}_{h}$ and $\mathscr{I}_{k}$, are refinements of the white noise regularization partitions $\mathscr{D}_{\sigma}$ and $\mathscr{I}_{\rho}$, respectively. For each $D_{m}^{\prime} \in \mathscr{D}_{h}$ there exists $D_{l} \in \mathscr{D}_{\sigma}$ such that $D_{m}^{\prime} \subset D_{l}$ etc.; this determines a unique mapping $\mu:\left[0: M^{\prime}\right] \rightarrow[0: M]$ such that $D_{m}^{\prime} \subset D_{\mu(m)}$. For simplicity, we also assume that the partitions are uniform and that the meshsize and timestep are denoted respectively by $h$ and $k$. The reason we do not make these partitions coincide is that for the finite element method's convergence analysis it may prove useful to have more involved couplings of the type $h=h(\sigma)$ and $k=k(\rho)$. In this article we consider only the simplest situation possible where $h=\sigma$ and $k=\rho$.

\subsection{Finite element space and the discrete scheme}

Let $\mathbb{V} \subset \mathrm{H}_{0}^{1}(D)$ be the space of continuous piecewise linear functions associated with the partition $\mathscr{D}_{h}$. We define the (spatial) semi-discrete solution as the time-dependent random finite element function $U:[0, T] \times \Omega \rightarrow \mathbb{V}$ which solves the SDE

$$
\left\langle\partial_{t} U(t), V\right\rangle+\left\langle\partial_{x} U(t), \partial_{x} V\right\rangle+\left\langle f_{\epsilon}(U(t)), V\right\rangle=\left\langle\partial_{x t} \bar{W}, V\right\rangle, \quad \forall V \in \mathbb{V}, t \in[0, T] .
$$

We discretize further this SDE in the time variable by taking a semi-implicit Euler scheme in time associated to the partition $I=\left\{t_{0}\right\} \cup \bigcup_{m} I_{m}$,

$$
\left\langle\frac{U^{n}-U^{n-1}}{k}, V\right\rangle+\left\langle\partial_{x} U^{n}, \partial_{x} V\right\rangle+\left\langle f_{\epsilon}\left(U^{n-1}\right), V\right\rangle=\left\langle\partial_{x t} \bar{W}, V\right\rangle, \quad \forall V \in \mathbb{V}, t \in[0, T]
$$

The scheme is semi-implicit in that the linear part is treated implicitly while it is explicit in the nonlinearity. This means that at each timestep only one linear problem has to be solved and no nonlinear solver is needed.

In practice, it is more practical to use a modified version of 6.3 given by

$$
\begin{aligned}
& \left\langle\frac{U^{n}-U^{n-1}}{k}, V\right\rangle+\left\langle\partial_{x} U^{n}, \partial_{x} V\right\rangle+\left\langle f_{\epsilon}^{\prime}\left(U^{n-1}\right) U^{n}, V\right\rangle \\
& \quad=\left\langle f_{\epsilon}^{\prime}\left(U^{n-1}\right) U^{n-1}-f_{\epsilon}\left(U^{n-1}\right), V\right\rangle+\epsilon^{\gamma}\left\langle\partial_{x t} \bar{W}, V\right\rangle, \quad \forall V \in \mathbb{V}, t \in[0, T],
\end{aligned}
$$

which allows bigger timesteps $k$ [KNS04]. Note that this amounts to a linearization involving one step of the Newton method to solve the nonlinear (fully implicit) backward Euler scheme. 


\subsection{The linear time-stepping system}

Let us indicate the basis functions of $\mathbb{V}$ by $\Phi_{m}$, for $m \in\left[0: M^{\prime}\right]$; these are the piecewise linear continuous functions such that $\Phi_{m}\left(x_{l}\right)=\delta_{l}^{m}$ for $l \in\left[0: M^{\prime}\right]$. If we indicate by $\boldsymbol{u}^{n}=\left(u_{m}^{n}\right)$ the vector of nodal values corresponding to the discrete solution $U^{n}$ at time $t^{n}$, that is, $U^{n}(x)=$ $\sum_{m=0}^{M^{\prime}} u_{m}^{n} \Phi_{m}(x)$, then we can translate 6.4 into the following matrix form:

$$
\left[\frac{1}{k} \boldsymbol{M}+\boldsymbol{A}+\frac{1}{\epsilon^{2}} \boldsymbol{N}\left(\boldsymbol{u}^{n-1}\right)\right] \boldsymbol{u}^{n}=\frac{1}{\epsilon^{2}} \boldsymbol{g}\left(\boldsymbol{u}^{n-1}\right)+\frac{1}{k} \boldsymbol{M} \boldsymbol{u}^{n-1}+\epsilon^{\gamma} \boldsymbol{w},
$$

where $\boldsymbol{M}, \boldsymbol{A}$ are the usual finite element mass and stiffness matrices, respectively, $\boldsymbol{N}\left(\boldsymbol{u}^{n-1}\right)$ and $\boldsymbol{g}\left(\boldsymbol{u}^{n-1}\right)$ are a "nonlinear" mass matrix and load vector, respectively, and $\boldsymbol{w}=\left(w_{m}\right)$ a random load vector generated at each timestep. A short calculation shows that for $m$ an internal degree of freedom (node) we have

$$
w_{m}=\frac{h}{2 \sqrt{\sigma \rho}}\left(\eta_{\mu(m)-1}+\eta_{\mu(m)}\right)
$$

where $\mu$ is the mapping introduced earlier in this section and $\eta_{l}$ is an $\mathrm{N}(0,1)$ random number for $l \in[0: M]$, or zero for $l=-1, M+1$ (the boundary cases). If the partitions $\mathscr{D}_{\sigma}$ and $\mathscr{D}_{h}$ coincide, which will be the case in the next section, then $h=\sigma$ and 6.6 simplifies to

$$
w_{m}=\frac{1}{2} \sqrt{\frac{h}{\rho}}\left(\eta_{m-1}+\eta_{m}\right) .
$$

This is the form that we employ in our computations below. From now on, we will assume $\mathscr{I}_{\rho}$ and $\mathscr{I}_{k}$ to coincide, i.e., $k=\rho$ in all our computations.

\section{Computations}

We now discuss numerical simulations of the Allen-Cahn problem, using the scheme (6.5). Our main purpose is to match the behavior of the exact stochastic solution with the behavior of the Monte Carlo type numerical solution. By "exact behavior" we refer to the theoretical results of Funaki [Fun95] and Brassesco et al. [BDMP95]. Our numerical experiments are directed towards testing relations between the various parameters $\epsilon, \gamma$ and $\rho, \sigma$. In addition to taking $k=\rho$, we also take $h=\sigma$.

\subsection{Monte Carlo simulations}

We use the finite element scheme 6.4 in combination with Monte Carlo type simulations. For each choice of parameters $\epsilon, \gamma$ and meshsize $h$, we choose the timestep $k=h^{2}$ and compute between 1000 and 2500 sample paths, each with a different seed for the pseudo-random number generator.

Each sample path runs from time 0 to a final time $T$. At the beginning of each run, the random number generator is seeded and the subsequent $\eta_{m}$ appearing in (6.7) are chosen according to this seed for all the run. The seed for each sample path is determined by the clock of the machine at the start of each run (these are also recorded for rerunning purposes). In this section we denote the numerical solution (which tacitly depends on $\epsilon, \gamma, h$, etc.) by $\left(U_{\omega}^{n}\right)_{n \in[0: N]}$, where 
$n$ corresponds to the timestep and $\omega$ is a discrete sample, i.e., the choice of the initial seed. We indicate by $\bar{\Omega}$ the discrete sample space, which can be thought of as being the set of all initial seeds employed.

\subsection{Phase-separation interfaces and centers}

Our benchmarking procedure consists in comparing the behavior of the center of the discrete solution with that of the exact solution. A function $v \in \mathrm{H}^{1}(D)$ is said to have a center if $v(x)=0$ is uniquely solvable with a sign change for $x \in D=(-1,1)$; the solution of $v(x)=0$ is called the center of $v$. For example the function $u_{0}(x)=\tanh \left(\left(x-x_{0}\right) / \sqrt{2} \epsilon\right)$ has center $x_{0}$.

The center represents a 0 -dimensional interface separating two phases in the 1-dimensional Allen-Cahn model considered here. Due to the noise a solution with center can nucleate, i.e., give rise to new zeroes, during the evolution; in this case it makes no longer sense to speak of "the" center and we simply say interface position. Such solutions, as we shall see, must be treated carefully in the statistics. We also note that an interface may disappear, either by exiting the domain $D$ or by annihilating another interface.

\subsection{A benchmark}

According to known results [Fun95, Theorem 8.1], [BDMP95] and the discussion done in 5.6. we expect the center of $U^{n} \in \mathrm{H}^{1}(D)$, if any, to perform a (discrete) Brownian motion, modulo perturbations of order $\mathrm{O}(\epsilon)$ and the numerical discretization error. Therefore, we will declare our numerical scheme to be acceptable if we see evidence of this Brownian motion in our computational results.

Based on this observation, our benchmarking procedure consists in tracking the center $\left(\Xi_{\omega}^{n}\right)_{0 \leqslant n \leqslant T / k} \subset(-1,1)$ of the computed sample path $\left(U_{\omega}^{n}\right)_{0 \leqslant n \leqslant T / k}$, where $\omega$ ranges over a discrete sample space, and we perform statistics on $\left(\Xi_{\omega}^{n}\right)_{0 \leqslant n \leqslant T / k}$ by averaging over $\omega$. In order to obtain meaningful statistics, we keep the number of interfaces constrained to 1. Paths that maintain a center up to the final time are called admissible sample paths. If new interfaces are created, or the center exits from $(-1,1)$ during the computation of one sample path, it is rejected as not admissible, the computation is stopped, and the computation of a new sample path is started. The average and variance of the interface position are computed over the admissible sample paths. The resulting average interface position, $\mathbb{E}\left[\Xi^{n}\right]$, and its variance, $\operatorname{Var}\left[\Xi^{n}\right]=\mathbb{E}\left[\left(\Xi^{n}\right)^{2}\right]-\left(\mathbb{E}\left[\Xi^{n}\right]\right)^{2}$, are realvalued functions of (discrete) time $t_{n}$.

According to $[5.40)$ and the subsequent remarks, the average position $\mathbb{E}\left[\Xi^{n}\right]$ must be close to 0 and its variance must be close to $c_{0} \epsilon^{1+2 \gamma} t_{n}$, and this is what we will be after in the next section.

Note that our use of the benchmark is not as strict as is usual in the context of numerical schemes for deterministic nonlinear equations. In the latter case, an exact solution is usually readily obtained and the benchmark procedure simply consists in measuring the error between the two solutions. In the linear stochastic case, a similar approach can be used with the moments of the SPDE's solution [ANZ98]. We should stress, however, that in our case, which is both stochastic and nonlinear, the exact solution is seldom known analytically, making such simple benchmarking nearly impossible.

\subsection{Simulations and results}

We run a series of Monte Carlo tests with various combination of parameters as follows: 


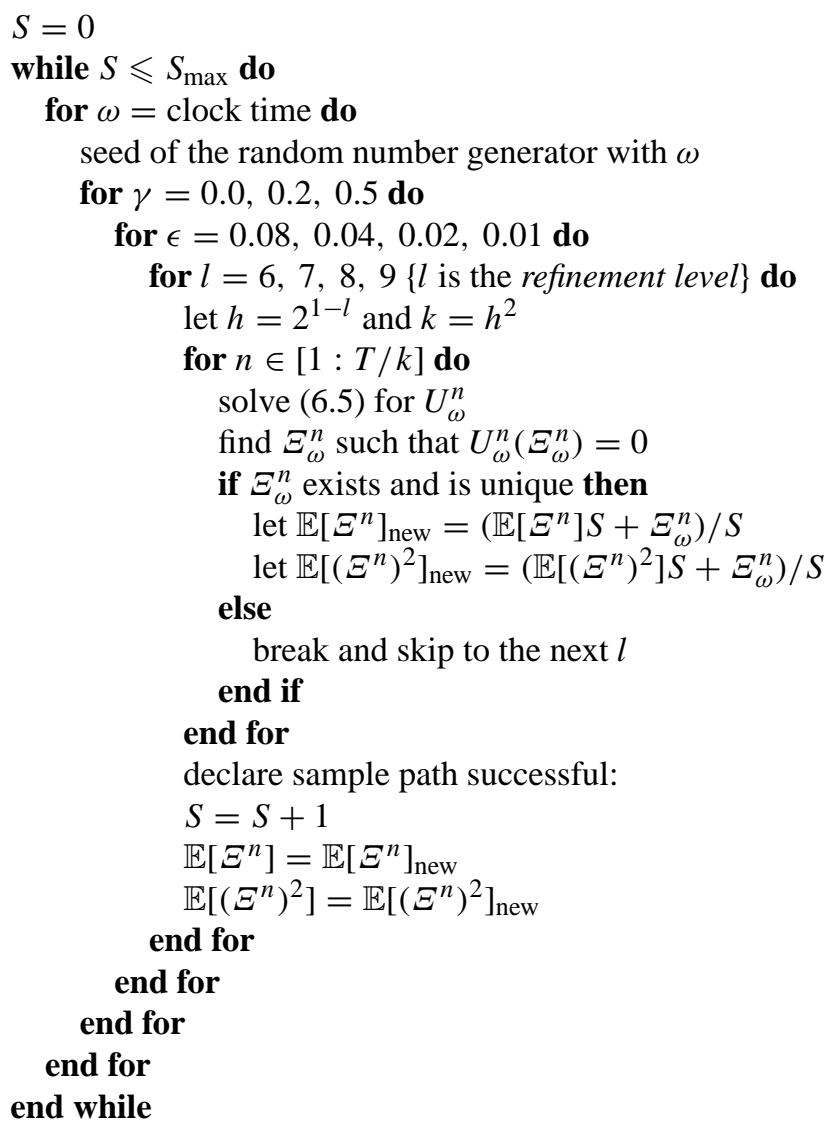

The results are reported in Figures 1, 2 and 3 for the values of $\gamma=0.0,0.2,0.5$, respectively. In each figure the sub-figures (a), (b) and (c), each of which is split into a top and bottom part, shows the graph, as (discrete) functions of $t_{n} \in[0, T]$, of the (discrete) average position $\mathbb{E}\left[\Xi^{n}\right]$ in the top part and its variance $\operatorname{Var} \Xi^{n}:=\mathbb{E}\left[\left(\Xi^{n}\right)^{2}\right]-\mathbb{E}\left[\Xi^{n}\right]^{2}$ rescaled by $1 / \epsilon^{1+2 \gamma} T$ in the bottom part for the values of $\epsilon=0.04,0.02,0.01$, respectively. (For easier visualization we plot the piecewise linear interpolation of discrete functions.) Different lines in each of these three diagrams correspond to different values of the mesh refinement level $l$. The absence of a line means that the total number of successful sample paths is 0 and no statistics are produced. The diagrams (a), (b), (c) correspond, in each figure, to the values of $\epsilon=0.04,0.02,0.01$. The plot $(\mathrm{d})$ is designed to see how well the scheme captures the $c_{0} \epsilon^{1+2 \gamma} t$ behavior of the variance; each line corresponds to a chosen but fixed value of $t$, and is a $\log -\log$ plot of the variance for the finest refinement level $(l=9)$ against $\epsilon$; different lines correspond to different times between 0 and $T=20$. For comparison we plot the line with slope $1+2 \gamma$, which represents the scaling power; lines parallel to this line mean that the code picks up the right scaling.

We next summarize the observations we have drawn from the computational results described.

Interface motion. The motion of the numerical interface, for sufficient transition layer and noise resolution, has the properties predicted by the analytical results. Indeed, starting from a 

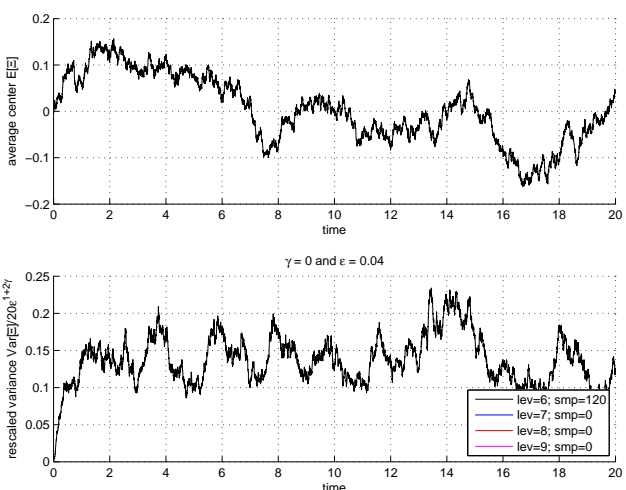

(a) Interface average (top), $\mathbb{E}\left[\Xi^{n}\right]$, and its rescaled variance (bottom), $\operatorname{Var}\left[\Xi^{n}\right] / 20 \epsilon^{1+2 \gamma}$, as functions of time $t_{n}$, for $\epsilon=0.04$. Only one line, corresponding to the coarsest refinement level $l=6$, i.e., meshsize $h=2^{-5}$, is visible as for all the other levels no admissible paths survive up to time $T=20$ with a center (i.e., only one zero).
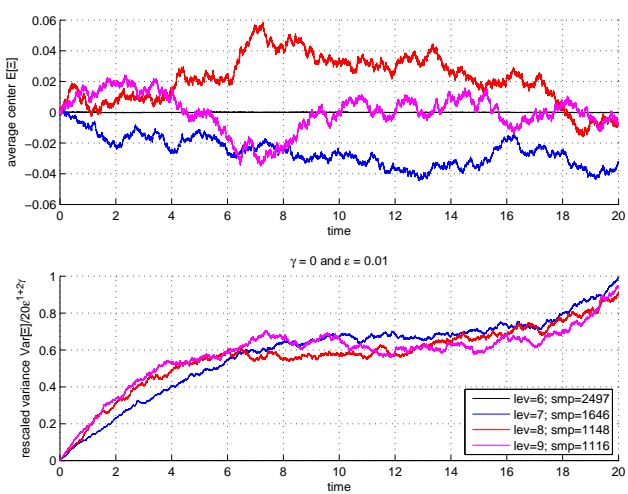

(c) Interface average (top), $\mathbb{E}\left[\Xi^{n}\right]$, and its rescaled variance (bottom), $\operatorname{Var}\left[\Xi^{n}\right] / 20 \epsilon^{1+2 \gamma}$, as functions of time $t_{n}$, for $\epsilon=0.01$. In each plot here, since $\epsilon$ is even smaller, we see four graphs corresponding to all four refinement levels tested, $l=6, \ldots, 9$. Note that the variance for $l=6$ is practically zero, which is far from the exact results. We believe that this is due to two concurrent factors: (1) the meshsize is too coarse to resolve the interface layer, (2) the meshsize is too coarse to resolve properly the noise and the numerical solution is closer to the exact deterministic solution rather than the stochastic one. Note also that the variance time-dependence, which is initially linear as expected, starts degenerating at about $T=4$.
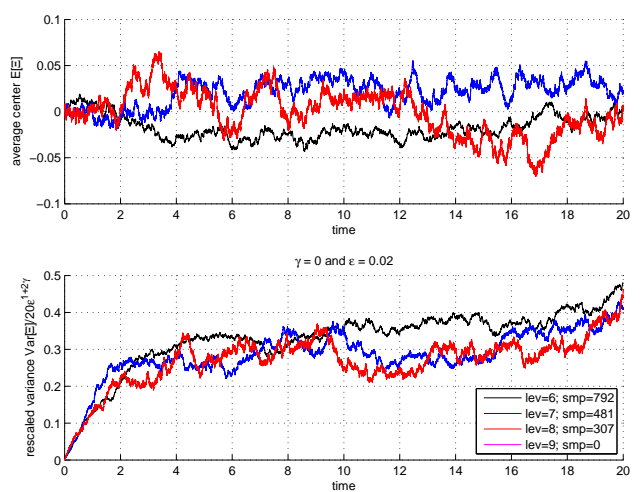

(b) Interface average (top), $\mathbb{E}\left[\Xi^{n}\right]$, and its rescaled variance (bottom), $\operatorname{Var}\left[\Xi^{n}\right] / 20 \epsilon^{1+2 \gamma}$, as functions of time $t_{n}$, for $\epsilon=0.02$. Comparing this with the case $\epsilon=$ 0.004 (Figure 1(a) we see that more graphs are visible in each diagram, corresponding to the refinement levels $l=6,7,8$. This is due to the fact that, as the value of $\epsilon$ decreases, the number of admissible paths increases.

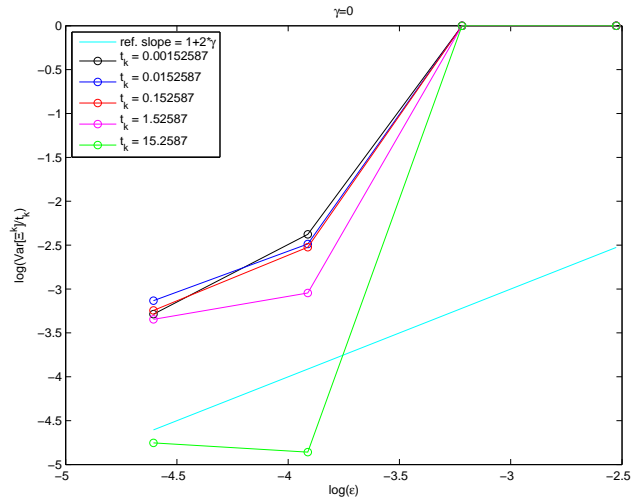

(d) These are the results from a different perspective, with a two-fold purpose: (1) to check the scaling power $1+2 \gamma$ in the diffusion coefficient, and (2) to see how the quality of results behaves with respect to time. Each of these graphs shows $\log \operatorname{Var}\left[\Xi^{n}\right]$ versus $\log \epsilon$ for the finest refinement level $l=9$. Each graph corresponds to a fixed choice of the discrete time index, $n$, from a sequence $n_{1}<\cdots<$ $n_{I}$, with $n_{i}=10 n_{i-1}$. A linear function of slope $1+2 \gamma$, which is the expected slope for the computed variances in the Mueller-Funaki time scale, is plotted for reference. We see that this is reflected by the computations, but with the quality worsening as time grows.

FIG. 1. Numerical results for $\gamma=0.0$ 

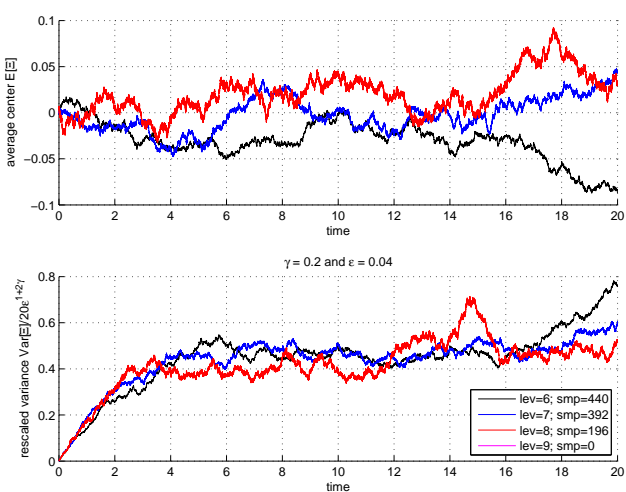

(a) Interface average (top), $\mathbb{E}\left[\Xi^{n}\right]$, and its rescaled variance (bottom), $\operatorname{Var}\left[\Xi^{n}\right] / 20 \epsilon^{1+2 \gamma}$, as functions of time $t_{n}$, for $\epsilon=0.04$. Compared to Figure 1(a) we see here that, due to the more modest intensity of the noise, three refinement levels produce enough admissible sample paths.
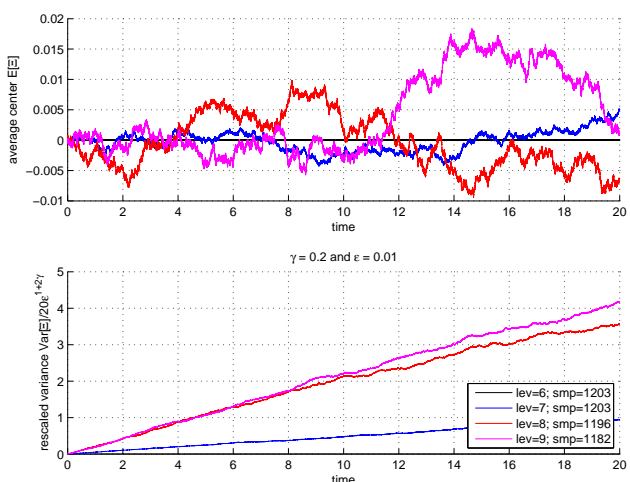

(c) Interface average (top), $\mathbb{E}\left[\Xi^{n}\right]$, and its rescaled variance (bottom), $\operatorname{Var}\left[\Xi^{n}\right] / 20 \epsilon^{1+2 \gamma}$, as functions of time $t_{n}$, for $\epsilon=0.01$. We see that the linear dependence on time is clearly visible in the variance, but for low refinement levels (coarse meshsize) the slope may not be the proper one. Also for very low refinement, the stochastic dynamics are not picked up at all.
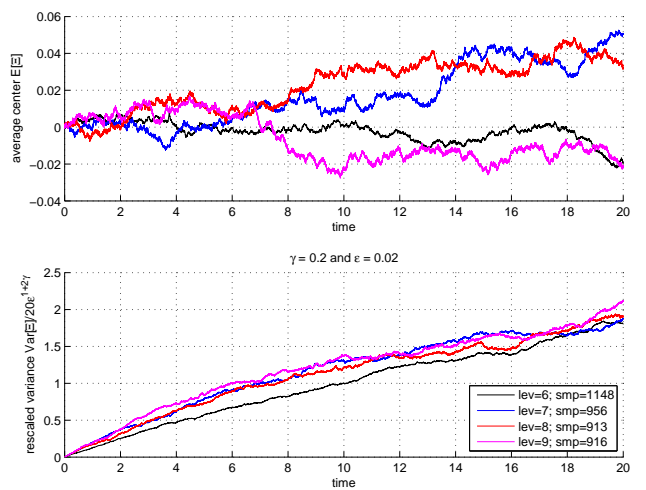

(b) Interface average (top), $\mathbb{E}\left[\Xi^{n}\right]$, and its rescaled variance (bottom), $\operatorname{Var}\left[\Xi^{n}\right] / 20 \epsilon^{1+2 \gamma}$, as functions of time $t_{n}$, for $\epsilon=0.02$. All four refinement levels are represented here. A clear linear trend in the computed variance, up to time 10 (with a minor deterioration between 10 and 20), is visible. This linear dependence is the expected behavior from the theory saying that $\operatorname{Var}\left[\Xi^{n}\right] \approx c_{0} \epsilon^{1+2 \gamma} t_{n}$.

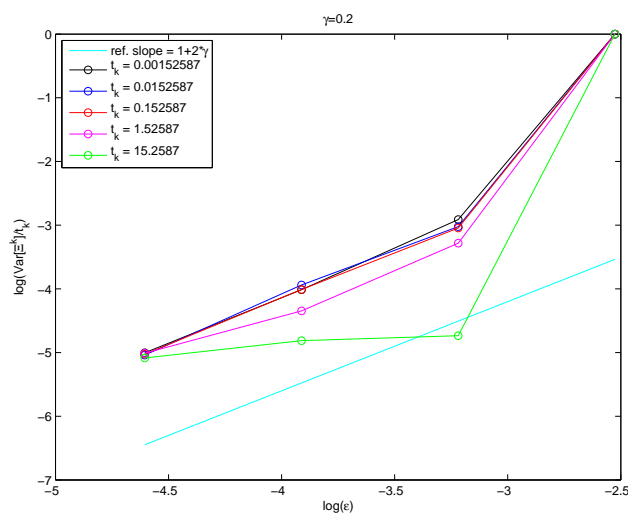

(d) Each of these graphs shows $\log \operatorname{Var}\left[\Xi^{n}\right]$ versus $\log \epsilon$ for the finest refinement level $l=9$. Each graph corresponds to a fixed choice of the discrete time index, $n$, from a sequence $n_{1}<\cdots<n_{I}$, with $n_{i}=10 n_{i-1}$. A linear function of slope $1+2 \gamma$, which is the expected slope for the computed variances in the Mueller-Funaki time scale, is plotted for reference. Comparing with the case $\epsilon=0.0$, an improvement in the quality of the behavior as $t_{n}$ grows is visible, but still a clear deterioration in time is present.

FIG. 2. Numerical results for $\gamma=0.2$. A comparison with Figure 1 is due and leads to two important observations. (1) The statistics are more robust in time (especially for $\epsilon$ small) in that the variance shows a clear linear dependence on $t$ in Figures 2(b) and 2(c) and the correct scaling behavior in Figure 2(d)] This is due to the lower intensity of the noise because $\gamma$ is bigger. (2) On the other hand, we must work harder with the refinement level in order to pick up the stochastic effects. We believe that this is a practical aspect of the discussion in $\$ 5.6$ This poor performance for bigger meshsizes could not be directly related to the interface layer resolution (a deterministic effect) as for the same values of $\epsilon$ and $h$ but lower values of $\gamma$ we obtain meaningful statistics, as shown in Figure 1 

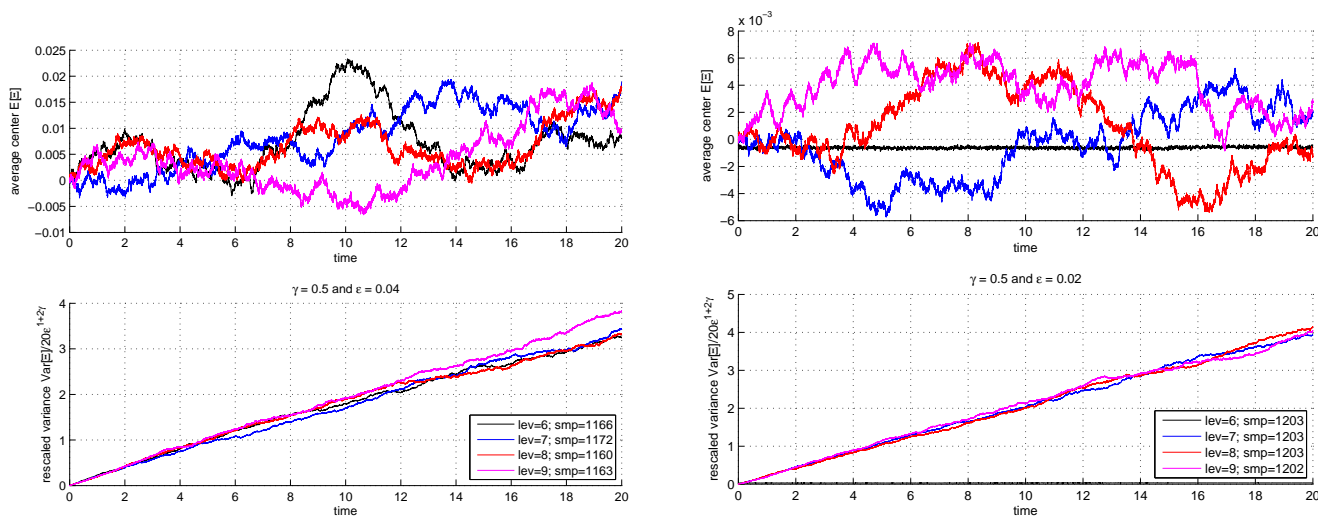

(a) Interface average (top), $\mathbb{E}\left[\Xi^{n}\right]$, and its rescaled variance (bottom), $\operatorname{Var}\left[\Xi^{n}\right] / 20 \epsilon^{1+2 \gamma}$, as functions of time $t_{n}$, for $\epsilon=0.04$.

(b) Interface average (top), $\mathbb{E}\left[\Xi^{n}\right]$, and its rescaled variance (bottom), $\operatorname{Var}\left[\Xi^{n}\right] / 20 \epsilon^{1+2 \gamma}$, as functions of time $t_{n}$, for $\epsilon=0.02$.
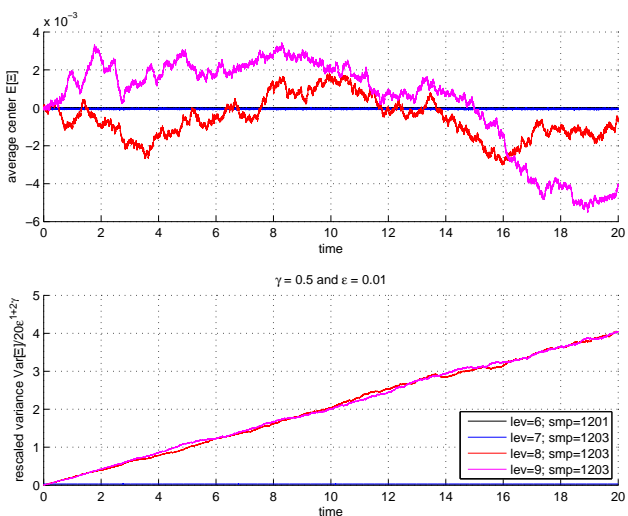

(c) Interface average (top), $\mathbb{E}\left[\Xi^{n}\right]$, and its rescaled variance (bottom), $\operatorname{Var}\left[\Xi^{n}\right] / 20 \epsilon^{1+2 \gamma}$, as functions of time $t_{n}$, for $\epsilon=0.01$. The linear time dependence of the (rescaled) variance is clear here. Note that for level of mesh refinement $l=6,7$ the computed solution is basically deterministic (average and variance are 0 ). This is a further indication of the practical importance of noise resolution, following the observations in $\$ 5.6$

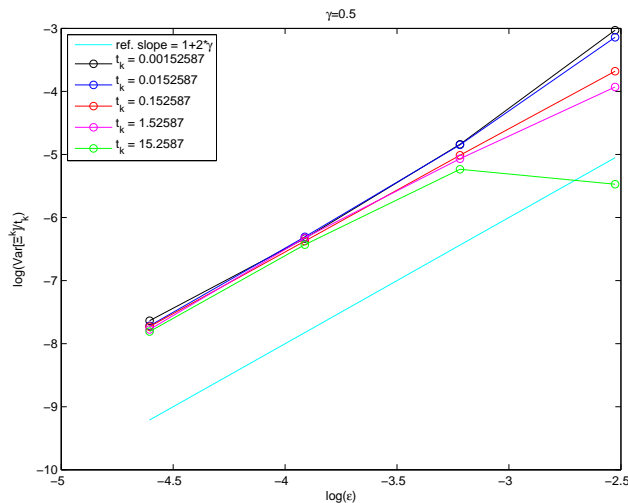

(d) Each of these graphs shows $\log \operatorname{Var}\left[\Xi^{n}\right]$ versus $\log \epsilon$ for the finest refinement level $l=9$. Each graph corresponds to a fixed choice of the discrete time index, $n$, from a sequence $n_{1}<\cdots<n_{I}$, with $n_{i}=10 n_{i-1}$ A linear function of slope $1+2 \gamma$, which is the expected slope for the computed variances in the Mueller-Funaki time scale, is plotted for reference. The value of $\gamma$ is quite high now as to keep the statistics robust with respect to time.

FIG. 3. Numerical results for $\gamma=0.5$. The same comments made in Figure 2apply here, but with an even clearer linear dependence of the variance upon time and a clearer need for high mesh refinement in order to resolve the white noise. Also the computations are more robust with respect to lower values of $\gamma$ and they show almost no deterioration in time.

resolved profile centered at zero, as seen in Figures 1,3 , the average position is near zero, whereas the variance, which is expected to be a linear function of time, behaves in accordance with the expectations, at least for some initial times; the $\gamma, \epsilon$ dependence of the diffusion coefficient $c_{0} \epsilon^{1+2 \gamma}$ is clearly captured by the numerics, as seen by the diagram (d) of each of the figures. 
Noise resolution. It is well known that for simulating the deterministic Allen-Cahn equation with any type of mesh/grid-based schemes, the meshsize has to be smaller than $\epsilon$ in order to resolve satisfactorily the transition layer about the interface. Intuitively, this is due to the fact that the transition layer has width $\mathrm{O}(\epsilon)$, and the numerical discretization parameters must be smaller than this width to have a proper resolution of this transition layer.

The effect of this is seen in each of Figures 1 to 3 as $\epsilon$ decreases, the level of refinement has to be taken bigger and bigger in order to obtain meaningful calculations.

In the stochastic case, the situation is complicated even more by the noise. Indeed, according to Theorem 5.4 and the discussion in \$5.6, the discretization parameters, in this case $h=\sigma$ and $k=\rho$, must be taken small enough to ensure that the noise effects are not lost for small $\epsilon$. Roughly speaking, the discretization parameters must be small, not only to resolve the interface, but also to resolve the noise and pick up the diffusion of the Brownian motion. This dependence, which is indicated analytically for $\gamma>3$ by Theorem 5.4, is also reflected in our computations for $\gamma \leqslant 3$. Indeed, a comparison between Figure 1 and Figure 2 shows how the level of refinement $l=7$ leads to meaningful results for $\gamma=0.0$ and all values of $\epsilon$ (at least for short times), whereas the same refinement level, for the same values of $\epsilon$, but with $\gamma=0.2$ is insufficient. For $\gamma=0.5$ in Figure 3 this phenomenon becomes yet more apparent.

We note that computations with $\gamma>3$ (not shown here) require an extremely fine mesh, and thus a very small timestep in view of Remark 4.6 , in order to capture any of the noise effects. Otherwise the deterministic solution will be computed. In this case, even choices of $h$ that resolve the interface satisfactorily are not enough to resolve the noise. The lesson we learn from this is that the interplay between the noise and the nonlinearity can be quite delicate, and not obviously predicted from deterministic considerations, in problems such as the stochastic Allen-Cahn equation.

Deterioration of simulations with $t$ big and $\epsilon$ small. As observed in the previous subsection, the computed variance depends linearly on time, as expected, but only for some initial time. The smaller $\epsilon$, the shorter this time is. This is seen in the bottom part of the sub-figures (a), (b) and (c) by the graph's earlier or later departure from an initial linear behavior. The computations deteriorate faster for small $\epsilon$, e.g., $\epsilon=0.0$, than they do for bigger $\epsilon$, e.g., $\epsilon=0.5$. The example with $\epsilon=0.2$ shows an intermediate behavior.

\subsection{Interface drift}

To conclude, we add some results of computations, for short times, with initial value a resolved profile centered away from 0 . In this case, the SDE describing the motion of the interface has also a drift term, which drives the interface towards the closest boundary of the domain. This drift is clearly seen for various choices of the parameters in the top diagrams of Figure 4 , where we plot the center's position average against time. Short times must be taken, for the statistics to make sense, otherwise solutions with centers that exit (or nucleate) cease to counterbalance those which stay in. Indeed, in diagram (c), where the noise intensity is quite strong, after an initial drift towards the boundary, the average inverts its route and moves away from the boundary. This is due to the fact that the statistics become too biased; the SE diagram shows the samples survival with respect to time (that is, a discrete-probability space version of the exit-time inverse function). The samples survival for the top diagrams is $100 \%$ for the statistics in the top diagrams. It is worth mentioning that similar observations using stochastic ODE's were made by Shardlow [Sha00]. 


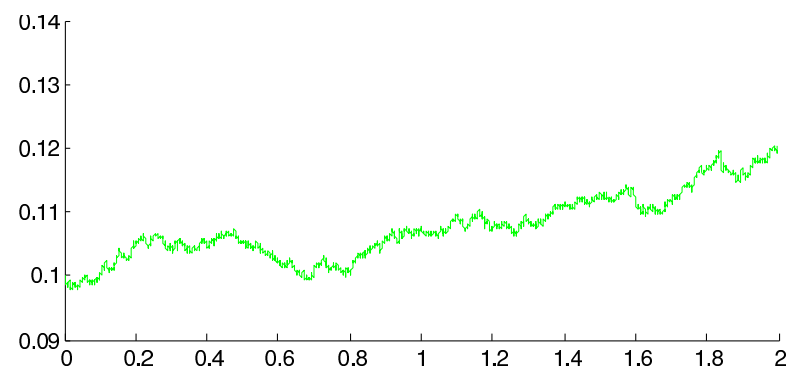

(a) The average of the interface position (center) for $\gamma=0.0$. An upward drift, that is, a drift of the interface towards the closest boundary point, is visible in this series of computations. The number of Monte Carlo samples is 1623. Only three of these paths violate the uniqueness of center condition and are therefore excluded from the statistics at the time when that happens.

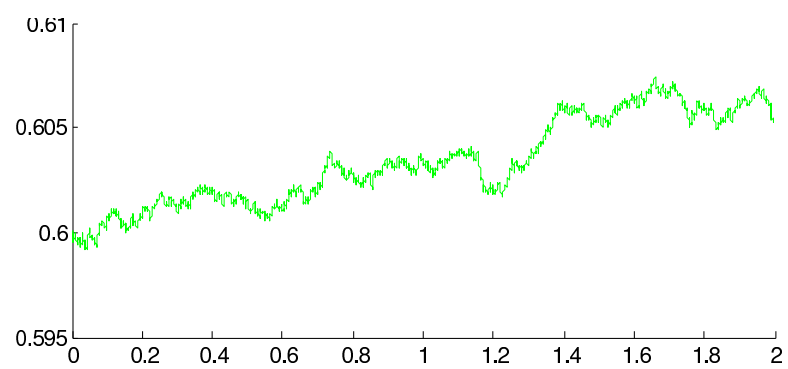

(b) The average of the interface position for $\gamma=0.2$. Compared with Figure 4(a) an outward drift is also detectable here, but due to the weaker noise intensity it is slower. The number of Monte Carlo samples here is 1752, all of which make it up to time $T=2$.
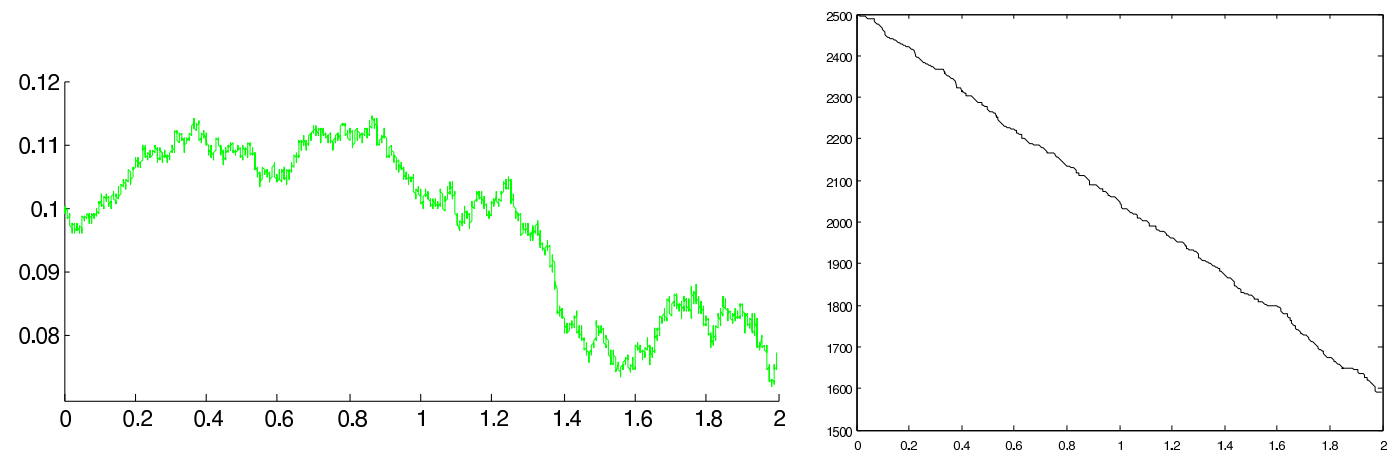

(c) On the left, we plot the average of the interface position for $\gamma=-0.1$. In this case, most sample paths do not make it with the condition of one interface, or center, up to time $T=2$. This fact is visualized on the right, where the curve represents the number of "admissible" sample paths (i.e., those that have a center) versus time; as time increases the number of admissible sample paths drops from an initial 2500 to almost 1500 . This high drop in the number of samples makes the statistics unreliable, and explains why the graph on the left exhibits no clear outward drift as a result.

FIG. 4. Monte Carlo computations of the drift effect for the initial interface position shifted away from zero. All these computations are performed for $\epsilon=0.01$, meshsize $h=2^{-8}$ and timestep $k=h^{2}$. See the text and Shardlow [Sha00], for example, for details about the drift. 


\section{Acknowledgments}

We would like to thank Georgios Zouraris and Anders Szepessy for stimulating discussions, and the anonymous referees for their interesting suggestions that led to the improvement of several aspects of this paper.

M.A.K.'s research is partially supported by the NSF grants DMS-ITR-0219211 and DMS0413864.

G.T.K.'s research is partially supported by the EU's Research Training Network on Fronts and Singularities HPRN-CT-2002-00274.

O.L. was supported by the EU's Research Training Network on Hyperbolic and Kinetic Equations (HYKE) HPRN-CT-2002-00282 and the EU's MCWave Marie Curie Fellowship HPMDCT-2001-00121 during his stay in Heraklion, and by The Nuffield Foundation's Young Researcher Grant in Brighton.

\section{REFERENCES}

[ANZ98] Allen, E. J., Novosel, S. J., \& Zhang, Z. Finite element and difference approximation of some linear stochastic partial differential equations. Stoch. Stoch. Rep. 64 (1998), 117-142. Zbl 0907.65147 MR 1637047

[AC79] ALlEN, S. M., \& CAHN, J. W. A macroscopic theory for antiphase boundary motion and its application to antiphase domain coarsening. Acta Metall. Mater. 27 (1979), 1085-1095.

[BTZ04] BABUŠKa, I., TEMpone, R., \& Zouraris, G. E. Galerkin finite element approximations of stochastic elliptic partial differential equations. SIAM J. Numer. Anal. 42 (2004), 800-825. Zbl 1080.65003 MR 2084236

[Bi195] Billingsley, P. Probability and Measure. 3rd ed., Wiley, New York (1995). Zbl 0822.60002 MR 1324786

[BPRS93] Bertini, L., Presutti, E., RÜDiger, B., \& SAada, E. Dynamical fluctuations at the critical point: convergence to a nonlinear stochastic PDE. Teor. Veroyatnost. i Primenen. 38 (1993), 689-741. Zbl 0819.60070 MR 1317994

[BDMP95] Brassesco, S., De Masi, A., \& Presutti, E. Brownian fluctuations of the interface in the $D=1$ Ginzburg-Landau equation with noise. Ann. Inst. H. Poincaré Probab. Statist. 31 (1995), 81-118. Zbl 0822.35158 MR 1340032

[Che94] Chen, X. F. Spectrum for the Allen-Cahn, Cahn-Hilliard, and phase-field equations for generic interfaces. Comm. Partial Differential Equations 19 (1994), 1371-1395. Z Zbl 0811.35098 MR 1284813

[Coo70] Cook, H. Brownian motion in spinodal decomposition. Acta Metall. 18 (1970), 297-306.

[DPZ92] DA PRAto, G., \& ZabCZYK, J. Stochastic Equations in Infinite Dimensions. Encyclopedia Math. Appl. 44, Cambridge Univ. Press, Cambridge (1992). Zbl 0761.60052 MR 1207136

[Doe87] DoERING, C. R. Nonlinear parabolic stochastic differential equations with additive colored noise on $\mathbb{R}^{d} \times \mathbb{R}_{+}$: A regulated stochastic quantization. Comm. Math. Phys. 109 (1987), 537-561. Zbl 0625.60069 MR 0885561

[DZ02] DU, Q., \& ZHANG, T. Y. Numerical approximation of some linear stochastic partial differential equations driven by special additive noises. SIAM J. Numer. Anal. 40 (2002), 1421-1445. Zbl 1030.65002 MR 1951901

[Dur99] DurRetT, R. Stochastic spatial models. SIAM Rev. 41 (1999), 677-718. Zbl 0940.60086 MR 1722998

[FJL82] FARIS, W. G., \& JONA-LASINIO, G. Large fluctuations for a nonlinear heat equation with noise. J. Phys. A 15 (1982), 3025-3055. Zbl 0496.60060 MR 0684578 
[FP03] FEnG, X., \& Prohl, A. Numerical analysis of the Allen-Cahn equation and approximation for mean curvature flows. Numer. Math. 94 (2003), 33-65. Zbl 1029.65093 MR 1971212

[FW05] FEnG, X., \& WU, H. J. A posteriori error estimates and an adaptive finite element method for the Allen-Cahn equation and the mean curvature flow. J. Sci. Comput. 24 (2005), 121-146. Zbl pre02238739 MR 2221163

[Fun95] FUnAKI, T. The scaling limit for a stochastic PDE and the separation of phases. Probab. Theory Related Fields 102 (1995), 221-288. Zbl 0834.60066 MR 1337253

[Gyö99] GYÖNGY, I. Lattice approximations for stochastic quasi-linear parabolic partial differential equations driven by space-time white noise. II. Potential Anal. 11 (1999), 1-37. Zbl 0944.60074 MR 1699161

[HH77] Hohenberg, P. C., \& Halperin, B. I. Theory of dynamic critical phenomena. Rev. Mod. Phys. 49 (1977), 435-479.

[KK01] Katsoulakis, M. A., \& KHo, A. T. Stochastic curvature flows: asymptotic derivation, level set formulation and numerical experiments. Interfaces Free Bound. 3 (2001), 265-290. Zbl 0991.35115 MR 1843588

[KX95] Kallianpur, G., \& XIONG, J. Stochastic Differential Equations in Infinite-Dimensional Spaces. Inst. Math. Statist. Lecture Notes Monogr. Ser. 26, Inst. Math. Statist., Hayward, CA (1995). Zbl 0859.60050 MR 1465436

[KNS04] Kessler, D., Nochetto, R. H., \& Schmidt, A. A posteriori error control for the Allen-Cahn problem: Circumventing Gronwall's inequality. M2AN Math. Model. Numer. Anal. 38 (2004), 129-142. MR 2073933

[KM99] Kielhorn, L., \& MUthukumaR, M. Spinodal decomposition of symmetric diblock copolymer homopolymer blends at the Lifshitz point. J. Chem. Phys. 110 (1999), 4079-4089.

[LSU68] Ladyzhenskaya, O. A., Solonnikov, V. A., \& Ural'TSEVA, N. N. Linear and Quasilinear Equations of Parabolic Type. "Nauka", Moscow (1968) (in Russian). Zbl 0164.12302 MR 0241821

[LN03] LIN, J. W. B., \& NeELIN, J. D. Toward stochastic deep convective parameterization in general circulation models. Geophys. Res. Lett. 30 (2003), 1162-1165.

[Liu03] LIU, D. Convergence of the spectral method for stochastic Ginzburg-Landau equation driven by space-time white noise. Commun. Math. Sci. 1 (2003), 361-375. Zbl pre02247626 MR 1980481

[dMS95] De Mottoni, P., \& Schatzman, M. Geometrical evolution of developed interfaces. Trans. Amer. Math. Soc. 347 (1995), 1533-1589. Zbl 0840.35010 MR 1672406

[MT95] MülleR, C., \& TRIBE, R. Stochastic p.d.e.'s arising from the long range contact and long range voter processes. Probab. Theory Related Fields 102 (1995), 519-545. Zbl 0827.60050 MR 1346264 MR 1764701

[ST03] Schwab, C., \& TOdoR, R.-A. Sparse finite elements for elliptic problems with stochastic loading. Numer. Math. 95 (2003), 707-734. Zbl 1044.65085 MR 2013125

[Sha00] ShardLow, T. Stochastic perturbations of the Allen-Cahn equation. Electron. J. Differential Equations 2000, no. 47, 19 pp. Zbl 0959.60047

[Spo89] Spohn, H. Scaling Limits of Interacting Particle Systems. Springer, New York (1989).

[Wal86] Walsh, J. B. An introduction to stochastic partial differential equations. École d'été de probabilités de Saint-Flour, XIV-1984, Lecture Notes in Math. 1180, Springer, Berlin (1986), 265-439. Zbl 0608.60060 MR 0876085

[WB95] Warren, J. A., \& Boettinger, W. J. Prediction of dendritic growth and microsegregation in a binary alloy using the phase-field method. Acta Metall. Mater. 43 (1995), 689-703. 